\title{
A Model Behind the Standard Model
}

\author{
CHAN Hong-Mo \\ h.m.chan @ rl.ac.uk \\ Rutherford Appleton Laboratory, \\ Chilton, Didcot, Oxon, OX11 0QX, United Kingdom \\ TSOU Sheung Tsun \\ tsou@ maths.ox.ac.uk \\ Mathematical Institute, University of Oxford, \\ 24-29 St. Giles', Oxford, OX1 3LB, United Kingdom
}

\begin{abstract}
In spite of its many successes, the Standard Model makes many empirical assumptions in the Higgs and fermion sectors for which a deeper theoretical basis is sought. Starting from the usual gauge symmetry $u(1) \times s u(2) \times s u(3)$ plus the 3 assumptions: (A) scalar fields as vielbeins in internal symmetry space 1 , (B) the "confinement picture" of symmetry breaking [2, 3, (C) generations as "dual" to colour [4], we are led to a scheme which offers: (I) a geometrical significance to scalar fields, (II) a theoretical criterion on what scalar fields are to be introduced, (III) a partial explanation of why su(2) appears broken while $s u(3)$ confines, (IV) baryon-lepton number (B - L) conservation, (V) the standard electroweak structure, (VI) a 3-valued generation index for leptons and quarks, and (VII) a dynamical system with all the essential features of an earlier phenomenological model [4] which gave a good description of the known mass and mixing patterns of quarks and leptons including neutrino oscillations. There are other implications the consistency of which with experiment, however, has not yet been systematically explored. A possible outcome is a whole new branch of particle spectroscopy from $s u(2)$ confinement, potentially as rich in details as that of hadrons from colour confinement, which will be accessible to experiment at high energy.
\end{abstract}




\section{Introduction}

Despite its many successes, the Standard Model as it now stands gives one the impression of being but the consequence of a deeper theory yet to be divined. The many input parameters on which it depends together with the intricate structural details which have to be built into it are a little beyond what one would expect of a truly fundamental theory. At a deeper level, one can wonder of course why nature should opt for a gauge symmetry of $u(1) \times s u(2) \times s u(3)$ and not some other symmetry, or why there should be a gauge structure in the first place. But even if one takes this particular gauge structure for granted, one finds that one has still to add quite a number of ingredients mostly connected to the Higgs and fermion sectors which are seemingly extraneous to the gauge hypothesis. For example, to break the electroweak $s u(2)$ symmetry so as to fit the picture obtained from experiment, one introduces in the Standard Model an su(2) doublet of scalar fields, whose significance in the original gauge framework is a little obscure, which is a pity, for the bosonic sector of the framework is otherwise so geometrical. Furthermore, we have not been able to explain why of the two nonabelian symmetries, the electroweak symmetry su(2) should be broken, while the colour symmetry $s u(3)$ is not. In the fermion sector also, nature tells us that we have to introduce 3 generations for each of the 4 fermion species: $U$ type quark, $D$-type quark, charged leptons and neutrinos, and that we have to take left-handed fermions as $s u(2)$ doublets and right-handed fermions singlets, without us being able to fathom why she would want us to do so. Indeed, it is this our inability to answer the above questions in the Higgs and fermion sectors which forces on us the bulk of the twenty-odd independent parameters that have to be fed into the Standard Model. Furthermore, at the secondary, detail level, there are the no less intriguing questions why the observed fermion masses should be hierarchical, with values differing from generation to generation by orders of magnitude, and why the mixing angles between up and down fermion states be so different from case to case, ranging from order $10^{-3}$ for $V_{u b}$, say, to order unity for the oscillation of atmospheric and solar neutrino, which questions are also left unanswered in the present Standard Model. Any understanding of them will thus be not only aesthetically satisfying but also of practical value, however incomplete the understanding we may be able to achieve at the present stage.

To attempt answering these questions, one can try extending the theory by enlarging the symmetry as in grand unified and supersymmetric theories, or by increasing the number of space-time dimensions as in Kaluza-Klein 
type theories, and/or the dimensions of the fundamental object as well, as in strings and branes. The tendency of such attempts, however, is to increase the number of unknown parameters rather than to reduce it. An alternative is to forego for the moment the very ambitious vision of the above attempts, attractive though it may be, opting instead for economy, and try to answer the questions posed all within the framework of the Standard Model.

It is in this latter spirit that we have attempted here to construct, based on some simple and hopefully reasonable assumptions, a new "protogenic" model which will give the Standard Model as the result. The attempt builds on three ideas which have been suggested earlier in slightly different contexts. The first (A) is the proposal that frame vectors in the gauge symmetry space be promoted to fields so as eventually to play the role of Higgs fields, thus giving the latter a geometrical significance which they at present lack [5, 1]. The second (B) is an old idea of 't Hooft [2] and of Banks and Rabinovici [3], re-emphasized more recently by 't Hooft [6], that symmetry breaking in certain circumstances may be re-interpreted as the consequence of a confining theory. The third $(\mathrm{C})$ is the suggestion that the generation symmetry is in some sense dual to colour su(3) symmetry [5, 4], so that one obtains automatically 3 and only 3 generations of fermions. These three ideas, as we hope to show, can bring us quite close to constructing a protogenic model behind the Standard Model as desired. Besides, they can each lead to quite revolutionary changes in our general concepts, which we wish first now to outline.

(A) The idea that scalar fields appearing in gauge theories may have the geometrical significance of frame vectors in internal symmetry space is analogous to the familiar concept of vierbeins being introduced as dynamical variables in gravitation theory. It means that these scalar fields, having a specific geometrical function to discharge, are to be regarded as an integral part of the gauge structure, and not to be introduced or discarded at will to fit our interpretation of data or some other prejudice. Their existence and properties are to be determined by the gauge symmetries in the theory leaving us little room for choice. Whether they can function as Higgs fields to break the appropriate symmetries and give a realistic model of nature has yet to be seen, but if they do, then the Higgs mechanism as normally conceived say in electroweak theory will cease to be an input assumption and become just a consequence of an all-enveloping gauge concept.

(B) It was shown by 't Hooft and Banks and Rabinovici already in the late seventies that the electroweak theory which is usually conceived as a theory with a spontaneously broken $s u(2)$ gauge symmetry can equivalently 
be considered as a theory where the $s u(2)$ gauge symmetry confines; what is actually broken is a global $s u(2)$ symmetry which is associated with but is not the same as the original local gauge symmetry. If we accept the latter interpretation, then the difference between $s u(2)$ and $s u(3)$ in the Standard Model is not any more a matter of status, i.e. whether spontaneously broken or confining, but just a matter of degree, i.e. how deeply the symmetry is confined so as to be accessible or not to probing by present experiment. If that is the case then it is a matter to be understood in terms of the dynamics and is not to be regarded as part of the empirical input.

(C) The idea that fermion generations may in some sense be dual to colour introduces automatically into the theory exactly 3 generations of fermions, the existence of which therefore need no longer to be assumed. Besides, the supposition brings with it its own dynamical logic so that the parameters which characterise the 3 fermion generations can now appear as dynamical consequences and again be removed from the Standard Model as inputs from experiment, thus drastically reducing the number of empirical parameters. Indeed, previously, with a phenomenological model we called the Dualized Standard Model (DSM) built roughly along these lines, we were already able to reproduce correctly most of the fermion mass ratios and mixing parameters [7, 4].

One sees therefore these ideas can in principle go a long way towards our goal of reducing the degree of arbitrariness in the formulation and serve as a basis for the construction of a protogenic version to the Standard Model. However, whether they will be able, on being put to practice, to produce a model approximating nature is a question which can be answered only by carrying out the program explicitly. The following is an outline of the logic followed and conclusions obtained in our attempt, which are to be detailed in the succeeding sections.

The idea (A) as developed in [1] specifies to a large extent what scalar fields corresponding to frame vectors (called framons henceforth) are to be introduced for a given gauge symmetry. When supplemented by an appeal to economy, it leads for the gauge symmetry $u(1) \times s u(2) \times s u(3)$ to 2 sets of framons: a "weak" framon transforming as a doublet of $s u(2)$ and 3 "strong" framons transforming as triplets of $s u(3)$. As frame vectors, they give the orientation of the local frames with respect to some global reference frames, and hence carry with them indices referring to the global frames as well. Since physics should not depend on the choice of reference frames, it follows that one has invariance not only under the original local gauge symmetries but also under the global symmetries $\tilde{u}(1) \times \widetilde{s u}(2) \times \widetilde{s u}(3)$. This doubled invariance 
puts a stringent condition on the framon action, in particular on the framon self-interaction potential which, up to 4th order for renormalizability, is found to have a unique form depending on 7 real parameters. The part of the potential depending only on the "weak" framon is identical in form to the standard electroweak potential, that only on the "strong" framons is similar in form to the scalar potential in DSM, but there are additional terms linking the "weak" and "strong" sectors. The vacuum for this potential is degenerate and depends, by virtue of the linkage terms, on the orientation of the "weak" framon in $\widetilde{s u}(3)$ space.

Next, in the confinement picture of [2] and [3] adopted in (B), all the local symmetries $u(1) \times s u(2) \times s u(3)$ remain exact, and both nonabelian symmetries confine, with the confinement by $s u(2)$ much deeper than that by (colour) $s u(3)$. What are broken are the global symmetries $\widetilde{s u}(2)$ and $\widetilde{s u}(3)$, the first by the $u(1)$ gauge interaction, the second by the framon potential above via the linkage to the "weak" sector. Under the present experimental regime where one already probes routinely into hadronic structures (call this the standard model scenario), one needs consider only su(2) as confining. In that case, only su(2) singlets are observable and still appear as elementary. In particular, as in the electroweak theory treated in [2] and [3], the Higgs boson appears as the $s$-wave bound state by $s u(2)$ confinement of the "weak" framon with its conjugate, while the vector bosons $W^{ \pm}, Z-\gamma$ appear as $p$ wave bound states, and one recovers the standard electroweak theory as the result.

Introducing next as fundamental fermion fields the simplest representations, say $\psi(1,1), \psi(1,3), \psi(2,1), \psi(2,3)$, where the first number denotes the $s u(2)$ and the second the $s u(3)$ representation, one can form bound states from the last 2 with the "weak" framon by $s u(2)$ confinement, which, as in the electroweak theory treated in [2], 3], and [1, represent respectively the left-handed leptons and quarks. There is one difference, however, namely that here the "weak" framon carry indices referring to the global symmetries $\tilde{u}(1), \widetilde{s u}(2)$, and $\widetilde{s u}(3)$, and these are now transmitted to the fermion bound states, i.e the left-handed leptons and quarks. The conserved $\tilde{u}(1)$ charge is found to be the baryon-lepton number, the $\widetilde{s u}(2)$ symmetry broken by $u(1)$ is identified already in [2, 3] and [1] as up-down flavour, while $\widetilde{s u}(3)$, broken in a rather special manner to be outlined in the next paragraph, is to play the role of generations, as proposed in $(\mathrm{C})$.

The Yukawa coupling constructed to have the required invariance gives 
for the tree-level mass matrix for both leptons and quarks a factorized form:

$$
m=m_{T} \boldsymbol{\alpha} \boldsymbol{\alpha}^{\dagger}
$$

where $m_{T}$ depends on the fermion species, but $\boldsymbol{\alpha}$, a vector in $\widetilde{s u}(3)$ space coming from the "weak" framons, does not. The vector $\boldsymbol{\alpha}$, however, is coupled to the "strong" framons via the linkage terms in the framon potential. An examination of the dynamics in the strong sector to first perturbative order along the lines already performed in [8] for DSM shows that $\boldsymbol{\alpha}$ will rotate in generation space as the scale $\mu$ changes, of which rotation there is a fixed point at $\mu=0$ and another at $\mu=\infty$, and $\boldsymbol{\alpha}$ rotates away from the first fixed point towards the second as $\mu$ increases. Now these properties of the fermion mass matrix are exactly those that were found in earlier analyses

[7] to be essential and very likely sufficient to give a reasonable description of the fermion mass and mixing patterns observed in experiment. It seems thus hopeful that similar agreement with experiment as for DSM can be achieved here although this can only be confirmed by an explicit calculation, which is now being pursued.

In view of these results, we venture to conclude that one does seem to have gone some way towards what we called a protogenic model behind the Standard Model. There are several unanswered questions, and distinguishing predictions to be tested, which we reserve for the concluding section, including in particular a whole possible new field of particle spectroscopy to be opened up, perhaps at LHC, but if not, then when high enough experimental energies become available.

\section{The Fundamental Boson Fields}

We start with a theory with gauge symmetry $u(1) \times s u(2) \times s u(3)$. Here and throughout this paper, we denote a gauge symmetry by its gauge (Lie) algebra whenever there is no necessity to specify which among the locally isomorphic Lie groups is to be selected as the gauge group, so as to avoid questions of topology inessential for the problem at hand. By convention, lower case letters denote algebras and upper case letters groups.

We introduce first as usual for the 3 factors respectively the gauge fields $A_{\mu}(x), B_{\mu}(x), C_{\mu}(x)$ with the well-known geometric significance of connections in the corresponding principal bundles.

In addition, following the suggestion (A) in the Introduction, we shall introduce as part of the gauge structure Lorentz scalar fields having the 
geometric significance of frame vectors in internal symmetry space, which we call here framons. The concept that frame vectors or vielbeins can be introduced as dynamical variables is familiar already in gravity theory. It thus requires no great stretch of imagination to consider having them as dynamical variables in gauge theories as well. Indeed, if gauge structures were to be obtained by compactification of higher dimensions as they are in certain string and Kaluza-Klein type theories, then it would seem natural, perhaps even necessary, to introduce, along with the vierbeins of gravity, such framons for gauge theories also.

Let us first make clear what we mean by frame vectors and framons in each of the 3 simple factors of the symmetry $u(1) \times s u(2) \times s u(3)$ of present interest, repeating briefly here some considerations in [1] for completeness. For the $s u(N)$ symmetries, frame vectors can be taken as the column vectors $\phi^{\tilde{a}}$ of the matrix:

$$
\Phi=\left(\phi_{a}^{\tilde{a}}\right),
$$

which specifies the orientation of the local (x-dependent) $s u(N)$ frame with respect to some global ( $x$-independent) reference frame, where $a=1,2, \ldots, N$ which labels the rows refers to the local frame and $\tilde{a}=1,2, \ldots, N$ which labels the columns refers to the global reference frame. By definition then, $\Phi$ transforms by a fundamental representation of the local $s u(N)$ operating from the left, but by an anti-fundamental representation of a global $\widetilde{s u}(N)$ operating from the right, where the latter represents the effect on $\Phi$ by a change in the global reference frame.

As a transformation matrix between $2 s u(N)$ frames (i.e. the local and the global), $\Phi$ would satisfy, of course, the unitary constraints:

$$
\Phi^{\dagger} \Phi=1, \quad \operatorname{det}(\Phi)=1,
$$

which means, in particular, that the frame vectors $\phi^{\tilde{a}}$ would each be of unit length. As framon fields, however, we would want the components of $\phi^{\tilde{a}}$, in analogy to the vierbeins of gravity, to have the freedom to vary over all (in this case, complex) values, so that (2.2) can no longer be fully satisfied. We could, of course, allow the components of $\Phi$ to vary independently over all complex values and ignore the constraint (2.2) altogether, thus introducing all the $N^{2}$ complex or $2 N^{2}$ real components of $\Phi$ as independent field variables. But this would seem extravagant, since these components need not all be independent. In order to minimize, for the sake of economy, the total number of independent fields to be introduced, we propose instead that we retain as much of (2.2) as is consistent with the desired freedom and with the natural condition that all framons have the same physical dimension. 
For $s u(2)$, we see that we can retain from (2.2) the condition:

$$
\phi_{r}^{\tilde{2}}=-\epsilon_{r s}\left(\phi_{s}^{\tilde{1}}\right)^{*},
$$

namely $\phi^{\tilde{1}}$ and $\phi^{\tilde{2}}$ being orthogonal and having the same length, but still allow all their components the freedom to range over all complex values. Besides, the relation being linear and homogeneous in $\phi^{\tilde{1}}$ and $\phi^{\tilde{2}}$ guarantees that they can remain of the same physical dimension. This means that for $s u(2)$ theory, we can eliminate via (2.3) one of the 2 framon fields in terms of the other and be left with only one independent $s u(2)$ doublet, say $\phi=\phi^{\tilde{1}}$, i.e. 2 complex or 4 real components as independent field variables, halving the number of the original components in $\Phi$. The same tactic, however, would not work for $s u(3)$, nor indeed for any $s u(N)$ with $N>2$. We could of course also reduce the number of independent framons in $s u(3)$ by insisting that the vector $\phi^{\tilde{3}}$, say, be always orthogonal to $\phi^{\tilde{1}}$ and $\phi^{\tilde{2}}$ by retaining from (2.2) the condition:

$$
\phi_{r}^{\tilde{3}}=\epsilon_{r s t}\left(\phi_{s}^{\tilde{1}}\right)^{*}\left(\phi_{t}^{\tilde{2}}\right)^{*},
$$

but this relation is inhomogeneous, implying for $\phi^{\tilde{3}}$ a different physical dimension from that of $\phi^{\tilde{1}}$ and $\phi^{\tilde{2}}$, a conclusion we cannot physically accept. The best that we can do, it seems, is just to retain from (2.2) the condition that the determinant be real. When cast in the form:

$$
\operatorname{det}(\Phi)=(\operatorname{det}(\Phi))^{*},
$$

the condition is homogeneous, thus not suffering from the objection against (2.4) above, and multilinear, thus allowing the independent variables to attain all values as demanded. This removes only one real component from the original 18 in $\Phi$ but seems already the best economy that can be achieved in $s u(3)$. As will be seen, this difference between $s u(2)$ and $s u(3)$, due just to their different structures, will play a significant role in this scheme in reproducing the very different physics arising from the 2 nonabelian symmetries in the Standard Model.

Next, let us repeat the above considerations for the remaining $u(1)$ factor. Here orientation means just a phase, and relative orientation just a phase difference. Hence, the analogue of $\Phi$ above for the $s u(N)$ factors is here just a phase factor of the form:

$$
\Phi=\exp i g_{1}(\alpha-\tilde{\alpha}),
$$


with $\alpha x$-dependent, transforming under the local $u(1)$ but $\tilde{\alpha} x$-independent, transforming under the global $\tilde{u}(1)$ transformations. The framon field is then just a complex scalar field with its phase as in (2.6) above.

Notice that the framon fields so introduced, simply by virtue of their assigned geometric significance as frame vectors, have a special property not shared by the gauge fields $A_{\mu}, B_{\mu}$ and $C_{\mu}$ introduced before nor by the fermion fields yet to be introduced. Namely, apart from transforming under the local (gauge) transformation as other fields do, they carry in addition a global in$\operatorname{dex}(\tilde{a}$ for the nonabelian symmetries and $\tilde{\alpha}$ for $u(1))$ which transforms under global changes of the reference frame. Since physics should not be affected by the choice of reference frames, the action for framons should be invariant also under such global changes. As will be seen, this helps to restrict the form of the action and leads automatically to additional conserved quantum numbers, which are assigned in the present scheme to such otherwise unexplained quantities as the baryon-lepton number and the (fermion) generation index.

Having now specified what is meant by framons for each of the simple factors in the symmetry $u(1) \times s u(2) \times s u(3)$ of actual interest, we turn now to the physical problem itself. Let us first consider the electroweak sector characterized by the local symmetry $u(1) \times s u(2)$ and ask here what framons should be introduced. We start again with a matrix giving the orientation of the local frame with respect to some global reference frame but now for the symmetry $u(1) \times s u(2)$. The columns of this matrix are what we call the frame vectors, which, as for the previous cases, are to be representations of the local symmetry $u(1) \times s u(2)$. Now, from the representations of the simple factor symmetries $u(1)$ and $s u(2)$, there are 2 ways to build a representation of $u(1) \times s u(2)$, i.e. taking either the sum or the product. Supposing we appeal again to economy and opt for the choice requiring the smallest number of independent framon fields, we would choose the product, since $1 \times 2<1+2$. In other words, the frame vectors would again be $s u(2)$ doublets, but now carrying each a $u(1)$ charge. Furthermore, by virtue of (2.3), we would eliminate one of these, leaving just one charged doublet, say, $\phi=\phi^{\tilde{1}}$ as the only framon field.

We have yet to specify what $u(1)$ charge this field $\phi$ should carry. This depends not just on the gauge algebra which is $u(1) \times s u(2)$, but on the choice of the gauge group. Now there are 3 locally isomorphic groups with this same algebra, namely: $U(1) \times S U(2), U(2)=(U(1) \times S U(2)) / Z_{2}$, and $U(1) \times S O(3)$, of which 3 we can discard immediately the last since it does not contain the $s u(2)$ doublet as a representation. Of the remaining 2 , the 
first, $U(1) \times S U(2)$, double-covers the second $U(2)$. Suppose we appeal again to minimality and choose the the smaller group $U(2)$, we end up with a halfintegral charge, say $\pm g_{1} / 2$, for the single doublet framon field $\boldsymbol{\phi}$. We notice that this is identical in properties to the Higgs field in standard electroweak theory. Indeed, starting with the framon concept, insisting on global $\tilde{u}(1) \times$ $\widetilde{s u}(2)$ invariance in addition to the usual local gauge $u(1) \times s u(2)$ invariance in constructing the action, and then following the above prescription based on minimality, one would be led uniquely to the standard electroweak theory as the result. A demonstration of this is given in [1] and again later in the full Standard Model context.

Having now applied the framon idea to the electroweak sector, let us push further and apply the same ideas to the full symmetry $u(1) \times s u(2) \times s u(3)$. We focus first on a single framon vector. This should be by itself a representation of the local symmetry $u(1) \times s u(2) \times s u(3)$, to be constructed out of the fundamental representations of the component symmetries, namely a phase for $u(1)$, a doublet for $s u(2)$, a triplet for $s u(3)$. For the product between each pair, one can take either the sum or the product representation. In the electroweak theory where the pair $u(1) \times s u(2)$ occurred, one took the product, this being the smaller of the two $(1 \times 2<1+2)$, minimising thus the number of scalar fields to be introduced. The same reasoning should apply to the product $u(1) \times s u(2)$ here, and also to $u(1) \times s u(3)$. For the pair $s u(2) \times s u(3)$, however, the sum representation is smaller than the product $(2 \times 3>2+3)$. Hence, following the same line of reasoning, to economize on the number of scalar fields, one would opt for the representation $\mathbf{1} \otimes(\mathbf{2} \oplus \mathbf{3})$. Practically, this means that each framon vector here is broken into 2 parts: a "weak" framon, $\phi_{r}, r=1,2$, being a doublet of $s u(2)$, and a "strong" framon, $\phi_{a}, a=1,2,3$, being a triplet of $s u(3)$, with each being also a representation of $u(1)$, i.e. carrying a $u(1)$ charge.

What $u(1)$ charges should these framons carry? By the symmetry $u(1) \times$ $s u(2) \times s u(3)$, we mean usually the algebra which, for constructing the action, for example, is all that matters. To identify what charges are permissible, however, we need to specify the gauge group [9]. Again, a version of this question in the electroweak theory was answered above correctly by invoking minimality. Suppose one takes the same attitude for the symmetry $u(1) \times$ $s u(2) \times s u(3)$; one would then adopt as gauge group the group, denoted $U(1,2,3)$ say, which is obtained by identifying in the group $U(1) \times S U(2) \times$ $S U(3)$ the following sextet of elements,

$$
(y, f, c)=\left(z^{4} y, f, \omega c\right)=\left(z^{2} y, f, \omega^{2} c\right)
$$




$$
=\left(z^{3} y,-f, c\right)=(z y,-f, \omega c)=\left(z^{5} y,-f, \omega^{2} c\right)
$$

where $y, f, c$, are elements in respectively in the groups $U(1), S U(2)$, and $S U(3)$

$$
z=\exp \pi i / 3, \quad \omega=\exp 2 \pi i / 3 .
$$

Note that $( \pm 1)$ and $\left(1, \omega, \omega^{2}\right)$ can respectively be identified with the elements of the centre of the groups $S U(2)$ and $S U(3)$. Being 6-fold covered by the group $U(1) \times S U(2) \times S U(3), U(1,2,3)$ is the "smallest" group with algebra $u(1) \times s u(2) \times s u(3)$ which admits as representations both the $\mathbf{2}$ of $s u(2)$ and the 3 of $s u(3)$. Then, with $U(1,2,3)$ specified as the gauge group, it follows that any field in the theory, which has to be a representation of $U(1,2,3)$, can have only the $u(1)$ charges $g_{1} q$, with $q$ depending on the representations in $s u(2)$ and $s u(3)$ as follows [9]:

$$
\begin{array}{ll}
(1,1) ; \quad & q=0+n, \\
(2,1) ; & q=\frac{1}{2}+n, \\
(1,3) ; & q=-\frac{1}{3}+n, \\
(2,3) ; & q=\frac{1}{6}+n,
\end{array}
$$

where the first number inside the brackets denotes the dimension of the representation in $s u(2)$ and the second number that in $s u(3)$, and $n$ can be any integer, positive or negative.

Restricting further the representations of the framons in $U(1,2,3)$ to those with minimal $|q|$, (i.e. equivalents in $U(1)$ of fundamental or antifundamental representations) one obtains then for the "weak" framon $q= \pm 1 / 2$ and for the "strong" framon $q=-1 / 3$. In consequence, they will acquire also the $\tilde{u}(1)$ charges $g_{1} \tilde{q}$ with $\tilde{q}$ opposite in sign to $q$, i.e. $\tilde{q}=\mp \frac{1}{2}$ for the "weak" framons, and $\tilde{q}=\frac{1}{3}$ for the "strong" framons.

We need more than one such framon, indeed as many framons as there are frame vectors for the symmetry $u(1) \times s u(2) \times s u(3)$. We recall that, as for the simple symmetries $s u(N)$ in (2.1) above, the "rows" of the present $\Phi$ matrix, i.e. the $(r, a)$-th components (for fixed $r, a)$ of the various frame vectors labelled by $(\tilde{r}, \tilde{a})$, and hence also of the framons, together should transform as a representation of the global symmetry $\tilde{u}(1) \times \widetilde{s u}(2) \times \widetilde{s u}(3)$. We thus have to ask again, which representation? Minimality would suggest again $\tilde{\mathbf{1}} \otimes(\tilde{\mathbf{2}} \oplus \tilde{\mathbf{3}})$, but if one chooses that, the theory would just break up into 2 separate theories, i.e. the electroweak theory plus chromodynamics disjoint from one another, which is not an interesting nor realistic situation. We propose therefore to opt instead for the all product representation $\tilde{\mathbf{1}} \otimes \tilde{\mathbf{2}} \otimes \tilde{\mathbf{3}}$. 
Although this apparently departs from the "principle of minimality" which has so far been our guideline, it does not affect the number of scalar $(x$ dependent) fields that have to be introduced which is still minimal since the symmetry under consideration is only global. In any case, if the proposal of the product representation $\tilde{\mathbf{1}} \otimes \tilde{\mathbf{2}} \otimes \tilde{\mathbf{3}}$ is accepted, we have finally for the full list of framon fields the following. For the "weak" framons, we have:

$$
\phi_{r}^{\tilde{r} \tilde{a}}=\alpha^{\tilde{a}} \phi_{r}^{\tilde{r}}, \quad r, \tilde{r}=1,2, \quad \tilde{a}=1,2,3, \quad q= \pm \frac{1}{2}, \quad \tilde{q}=\mp \frac{1}{2},
$$

and for the "strong" framons,

$$
\phi_{a}^{\tilde{r} \tilde{a}}=\alpha^{\tilde{r}} \phi_{a}^{\tilde{a}}, \quad \tilde{r}=1,2, \quad a, \tilde{a}=1,2,3, \quad q=-\frac{1}{3}, \quad \tilde{q}=\frac{1}{3} .
$$

The quantities $\alpha^{\tilde{r}}$ and $\alpha^{\tilde{a}}$ are independent of $x$, but $\phi_{r}^{\tilde{r}}$ and $\phi_{a}^{\tilde{a}}$ are $x$-dependent representing altogether $4+9=13$ complex scalar fields or 26 real fields. Because of the unitarity constraint (2.3) for $\phi_{r}^{\tilde{r}}$ and that the determinant be real for $\phi_{a}^{\tilde{a}}$, not all of these are actually independent, and one ends up for the framons with just 21 real field degrees of freedom.

The specific set-up (2.10) and (2.11) of framons is arrived at by insisting, when faced with ambiguities, on "minimality", which though appealing for economy is by no means compelling since one cannot as yet give a physical reason why nature should opt for economy. Indeed, we have to admit that in arriving at the above conclusion along the lines described, we have been peeping at the physical consequences also, and have no doubt been influenced by physical considerations. For example, in deciding on which representation a framon should take in the symmetry $u(1) \times s u(2) \times s u(3)$, we have tried at first the full product $\mathbf{1} \otimes \mathbf{2} \otimes \mathbf{3}$ but had to discard it for giving a physically inadmissible amount of mixing between the weak and strong sectors. Also, while opting for the group $U(1,2,3)$, we knew already from previous analysis that it is the group that nature seems to prefer. So, the above derivation through minimality is in a sense an afterthought. Nevertheless, we find it interesting that by appealing to minimality when faced with ambiguities left open by the framon idea, one is led almost uniquely (i.e. apart from the choice of representation for the global symmetry already mentioned) to the only solution which seems to work, namely the one set out in (2.10) and (2.11) that we shall henceforth adopt.

The framon fields in (2.10) and (2.11) are of a form unfamiliar at least to us, having as they do each an $x$-independent factor $\alpha$ which depends on symmetry indices. We find it convenient to picture them as rather like the nucleon wave function in nuclear physics when isospin is considered as 
an exact global symmetry. To describe there a nucleon, we have not only to give the wave function dependent on $x$ and on spin, but also to specify whether it is a proton or a neutron by an isospin factor independent both of $x$ and of spin.

For future use, we choose to normalize the $\alpha$ 's, as we are free to do, such that

$$
\sum_{\tilde{a}}\left|\alpha^{\tilde{a}}\right|^{2}=1, \quad \sum_{\tilde{r}}\left|\alpha^{\tilde{r}}\right|^{2}=1 .
$$

We shall find it convenient also to adopt on occasion a vector notation grouping various components of the framons, thus:

$$
\left(\phi^{\tilde{r}}\right)_{r}=\left(\phi_{r}\right)^{\tilde{r}}=\phi_{r}^{\tilde{r}}, \quad\left(\phi^{\tilde{a}}\right)_{a}=\left(\phi_{a}\right)^{\tilde{a}}=\phi_{a}^{\tilde{a}},
$$

where $\phi$ can denote a 2 -dimensional vector in either $s u(2)$ or $\widetilde{s u}(2)$ space, or a 3-dimensional vector in either $s u(3)$ or $\widetilde{s u}(3)$ space. Any ambiguity which might arise in this notation can readily be resolved by noting how the vectors are labelled and by the context.

For the weak framons $\phi_{r}^{\tilde{r} a}$, one can economize on the notation further by eliminating $\phi^{\tilde{2}}$ via (2.3) leaving only

$$
\alpha^{\tilde{a}} \phi_{r}=\alpha^{\tilde{a}}\left(\phi^{\tilde{1}}\right)_{r}
$$

where $\phi_{r}$ can be taken with a definite charge e.g. $\phi_{r}^{(-)}$as we shall sometimes do in future. However, to exhibit the $\widetilde{s u}(2)$ invariance of the theory, it is often more convenient to keep the more general notation with both $\tilde{r}=1,2$ where neither carries a definite $u(1)$ charge, thus:

$$
\phi_{r}^{( \pm)}=\alpha_{\tilde{1}}^{( \pm)} \phi_{r}^{\tilde{1}}+\alpha_{\tilde{2}}^{( \pm)} \phi_{r}^{\tilde{2}}=\boldsymbol{\alpha}^{( \pm)} \cdot \phi_{r},
$$

with

$$
\left|\boldsymbol{\alpha}^{(+)}\right|=\left|\boldsymbol{\alpha}^{(-)}\right|=1, \quad \boldsymbol{\alpha}^{(+)} \cdot \boldsymbol{\alpha}^{(-)}=0 .
$$

With the specification of the framon fields in addition to the gauge fields introduced at the beginning, our list of the fundamental boson fields is now complete.

\section{The Invariant Action}

Next, we turn to the construction of an invariant action for the bosonic fields enumerated above. Apart from Lorentz invariance, we shall require of 
course that the action be invariant under local transformations of the initial gauge symmetries $u(1) \times s u(2) \times s u(3)$. Furthermore, given that the global symmetries $\tilde{u}(1) \times \widetilde{s u}(2) \times \widetilde{s u}(3)$ which enter in the framon fields originate only as the choice of reference frames of which physics should presumably be independent, we ought to require also that our action be invariant under these as well. We shall therefore construct an action on this basis, which is fairly stringent, leaving rather little freedom for its choice.

As usual we write our action for the bosonic sector as a sum of 3 terms:

$$
\mathcal{A}_{B}=\mathcal{A}_{G F}+\mathcal{A}_{K E}+\int V
$$

with $\mathcal{A}_{G F}$ representing the free action for the gauge fields, and $\mathcal{A}_{K E}$ and $V$ respectively the kinetic energy and the potential of self-interaction for the scalar framons.

Explicitly, for $\mathcal{A}_{G F}$, we write as usual:

$$
\mathcal{A}_{G F}=-\frac{1}{4} \int d^{4} x F_{\mu \nu} F^{\mu \nu}-\frac{1}{4} \int d^{4} x \operatorname{Tr}\left(G_{\mu \nu} G^{\mu \nu}\right)-\frac{1}{4} \int d^{4} x \operatorname{Tr}\left(H_{\mu \nu} H^{\mu \nu}\right),
$$

with

$$
\begin{aligned}
F_{\mu \nu} & =\partial_{\nu} A_{\mu}-\partial_{\mu} A_{\nu} \\
G_{\mu \nu} & =\partial_{\nu} B_{\mu}-\partial_{\mu} B_{\nu}+i g_{2}\left[B_{\mu}, B_{\nu}\right], \\
H_{\mu \nu} & =\partial_{\nu} C_{\mu}-\partial_{\mu} C_{\nu}+i g_{3}\left[C_{\mu}, C_{\nu}\right],
\end{aligned}
$$

for respectively the $u(1), s u(2)$ and $s u(3)$ components. This action $\mathcal{A}_{G F}$ is of course constructed to be invariant under the local symmetries. It is invariant also under the global symmetries $\tilde{u}(1) \times \widetilde{s u}(2) \times \widetilde{s u}(3)$, trivially since on these it does not depend.

Next, for the kinetic energy term $\mathcal{A}_{K E}$ we write as usual:

$$
\mathcal{A}_{K E W}=\int \sum_{r, \tilde{r}, \tilde{a}}\left[\left(\partial_{\mu}-i g_{1} q A_{\mu}-i g_{2} B_{\mu}\right)_{r s} \phi_{s}^{\tilde{r} \tilde{a}}\right]^{*} \times[\text { h.c. }],
$$

for the weak framons $\phi_{r}^{\tilde{r} \tilde{a}}$, where $q$ denotes the $u(1)$ charge operator, and

$$
\mathcal{A}_{K E S}=\int \sum_{a, \tilde{r}, \tilde{a}}\left[\left(\partial_{\mu}+i \frac{g_{1}}{3} A_{\mu}-i g_{3} C_{\mu}\right)_{a b} \phi_{b}^{\tilde{r} \tilde{a}}\right]^{*} \times[\text { h.c. }],
$$


for the strong framons $\phi_{a}^{\tilde{r} \tilde{a}}$. These kinetic energy terms are by construction invariant under both the local and the global symmetries.

By virtue of the conditions (2.10), the weak framon term reduces to:

$$
\mathcal{A}_{K E W}=\int \sum_{\tilde{r}}\left(D_{\mu} \phi^{\tilde{r}}\right)^{\dagger}\left(D^{\mu} \boldsymbol{\phi}^{\tilde{r}}\right),
$$

with

$$
D_{\mu} \boldsymbol{\phi}^{\tilde{r}}=\left(\partial_{\mu}-i g_{1} q A_{\mu}-i g_{2} B_{\mu}\right) \boldsymbol{\phi}^{\tilde{r}}
$$

while by (2.11) the strong framon term reduces to:

$$
\mathcal{A}_{K E S}=\int \sum_{\tilde{a}}\left(D_{\mu} \phi^{\tilde{a}}\right)^{\dagger}\left(D^{\mu} \boldsymbol{\phi}^{\tilde{a}}\right),
$$

with

$$
D_{\mu} \boldsymbol{\phi}^{\tilde{a}}=\left(\partial_{\mu}+i \frac{g_{1}}{3} A_{\mu}-i g_{3} C_{\mu}\right) \boldsymbol{\phi}^{\tilde{a}} .
$$

Further, by virtue of the conditions (2.3) and (2.14), the kinetic energy term for weak framons reduces to just:

$$
\mathcal{A}_{K E W}=2 \int\left(D_{\mu} \boldsymbol{\phi}\right)^{\dagger}\left(D^{\mu} \boldsymbol{\phi}\right)
$$

which, if we choose $\phi=\phi^{(-)}$, is the same as in standard electroweak theory, apart from an unimportant factor 2 .

Lastly, to construct the general interaction potential for framons with the required invariance under both the local and global symmetries, we take a sum of all terms up to fourth order (for renormalizability) in the framon fields, contracting the indices in all possible ways. We obtain the following.:

$$
V[\Phi]=V_{W}[\Phi]+V_{S}[\Phi]+V_{W S}[\Phi]
$$

where $V_{W}$ involves only the weak framons, $V_{S}$ only the strong framons, and $V_{W S}$ both. Explicitly, for the weak sector, we have:

$$
\begin{aligned}
V_{W}[\Phi] & =-\mu_{W}^{\prime} \sum_{r, \tilde{r}, \tilde{a}} \phi_{r}^{\tilde{r} \tilde{a} *} \phi_{r}^{\tilde{r} \tilde{a}}+\lambda_{W}^{\prime}\left[\sum_{r, \tilde{r}, \tilde{a}} \phi_{r}^{\tilde{r} \tilde{a} *} \phi_{r}^{\tilde{r} \tilde{a}}\right]^{2}+\kappa_{1 W} \sum_{r, s, \tilde{r}, \tilde{s}, \tilde{a}, \tilde{b}} \phi_{r}^{\tilde{r} \tilde{a} *} \phi_{r}^{\tilde{r} \tilde{b}} \phi_{s}^{\tilde{s} \tilde{b} *} \phi_{s}^{\tilde{s} \tilde{a}} \\
& +\kappa_{2 W} \sum_{r, s, \tilde{r}, \tilde{s}, \tilde{a}, \tilde{b}} \phi_{r}^{\tilde{r} \tilde{a} *} \phi_{r}^{\tilde{s} \tilde{a}} \phi_{s}^{\tilde{s} \tilde{b} *} \phi_{s}^{\tilde{r} \tilde{b}}+\kappa_{3 W} \sum_{r, s, \tilde{r}, \tilde{s}, \tilde{a}, \tilde{b}} \phi_{r}^{\tilde{r} \tilde{a} *} \phi_{s}^{\tilde{r} \tilde{a}} \phi_{s}^{\tilde{s} \tilde{s} *} \phi_{r}^{\tilde{s} \tilde{b}},
\end{aligned}
$$


for the strong sector, we have:

$$
\begin{aligned}
V_{S}[\Phi] & =-\mu_{S}^{\prime} \sum_{a, \tilde{r}, \tilde{a}} \phi_{a}^{\tilde{r} \tilde{a} *} \phi_{a}^{\tilde{r} \tilde{a}}+\lambda_{S}^{\prime}\left[\sum_{a, \tilde{r}, \tilde{a}} \phi_{a}^{\tilde{r} \tilde{a} *} \phi_{a}^{\tilde{r} \tilde{a}}\right]^{2}+\kappa_{1 S} \sum_{a, b, \tilde{r}, \tilde{s}, \tilde{a}, \tilde{b}} \phi_{a}^{\tilde{r} \tilde{a} *} \phi_{a}^{\tilde{s} \tilde{a}} \phi_{b}^{\tilde{\tilde{s}} \tilde{b} *} \phi_{b}^{\tilde{r} \tilde{b}} \\
& +\kappa_{2 S} \sum_{a, b, \tilde{r}, \tilde{s}, \tilde{a}, \tilde{b}} \phi_{a}^{\tilde{r} \tilde{a} *} \phi_{a}^{\tilde{r} \tilde{b}} \phi_{b}^{\tilde{s} \tilde{b} *} \phi_{b}^{\tilde{s} \tilde{a}}+\kappa_{3 S} \sum_{a, b, \tilde{r}, \tilde{s}, \tilde{a}, \tilde{b}} \phi_{a}^{\tilde{r} \tilde{a} *} \phi_{b}^{\tilde{r} \tilde{a}} \phi_{b}^{\tilde{s} \tilde{b} *} \phi_{a}^{\tilde{s} \tilde{b}},
\end{aligned}
$$

and for the interaction between the two, we have:

$$
\begin{aligned}
V_{W S}[\Phi] & =\nu_{11} \sum_{r, a, \tilde{r}, \tilde{s}, \tilde{a}, \tilde{b}} \phi_{r}^{\tilde{r} \tilde{a} *} \phi_{r}^{\tilde{r} \tilde{a}} \phi_{a}^{\tilde{s} \tilde{b} *} \phi_{a}^{\tilde{s} \tilde{b}}+\nu_{21} \sum_{r, a, \tilde{r}, \tilde{s}, \tilde{a}, \tilde{b}} \phi_{r}^{\tilde{r} \tilde{a} *} \phi_{r}^{\tilde{r} \tilde{b}} \phi_{a}^{\tilde{s} \tilde{b} *} \phi_{a}^{\tilde{s} \tilde{a}} \\
& +\nu_{12} \sum_{r, a, \tilde{r}, \tilde{s}, \tilde{a}, \tilde{b}} \phi_{r}^{\tilde{r} \tilde{a} *} \phi_{r}^{\tilde{s} \tilde{a}} \phi_{a}^{\tilde{s} \tilde{s} *} \phi_{a}^{\tilde{s} \tilde{b}}+\nu_{22} \sum_{r, a, \tilde{r}, \tilde{s}, \tilde{s}, \tilde{a}, \tilde{b}} \phi_{a}^{\tilde{r} \tilde{a}} .
\end{aligned}
$$

Imposing now the conditions (2.3) and (2.14) eliminating thus $\phi_{r}^{\tilde{2}}$ in terms of $\phi_{r}^{\tilde{1}}=\phi_{r}$, we find that the $\kappa$ terms in $V_{W}$ are of the same form as the $\lambda$ term so that the whole of $V_{W}$ reduces to:

$$
V_{W}[\Phi]=-\mu_{W}|\phi|^{2}+\lambda_{W}\left(|\phi|^{2}\right)^{2},
$$

i.e. the same as in the standard electroweak theory, only with $\mu_{W}=2 \mu_{W}^{\prime}$, and $\lambda_{W}=4 \lambda_{W}^{\prime}+4 \kappa_{1 W}+2 \kappa_{2 W}+2 \kappa_{3 W}$. Imposing next the conditions (2.11) on $V_{S}$, we find that the $\kappa_{1 S}$ term becomes the same as the $\lambda_{S}$ term while the $\kappa_{2 S}$ and $\kappa_{3 S}$ terms take on the same form, so that the whole of $V_{S}$ reduces to:

$$
V_{S}[\Phi]=-\mu_{S} \sum_{a, \tilde{a}}\left(\phi_{a}^{\tilde{a} *} \phi_{a}^{\tilde{a}}\right)+\lambda_{S}\left[\sum_{a, \tilde{a}}\left(\phi_{a}^{\tilde{a} *} \phi_{a}^{\tilde{a}}\right)\right]^{2}+\kappa_{S} \sum_{a, b, \tilde{a}, \tilde{b}}\left(\phi_{a}^{\tilde{a} *} \phi_{a}^{\tilde{b}}\right)\left(\phi_{b}^{\tilde{b} *} \phi_{b}^{\tilde{a}}\right),
$$

which is the same as the framon potential for the pure $s u(3)$ theory given in [1], only with $\mu_{S}=\mu_{S}^{\prime}, \lambda_{S}=\lambda_{S}^{\prime}+\kappa_{1 S}$, and $\kappa_{S}=\kappa_{2 S}+\kappa_{3 S}$. Similarly, imposing both (2.10) and (2.11), one obtains that in $V_{W S}$, the $\nu$ terms are equal in form in pairs, giving:

$$
V_{W S}[\Phi]=\nu_{1}|\phi|^{2} \sum_{a, \tilde{a}} \phi_{a}^{\tilde{a} *} \phi_{a}^{\tilde{a}}+\nu_{2}|\phi|^{2} \sum_{a}\left|\sum_{\tilde{a}}\left(\alpha^{\tilde{a} *} \phi_{a}^{\tilde{a}}\right)\right|^{2},
$$

with $\nu_{1}=2 \nu_{11}+\nu_{12}$ and $\nu_{2}=2 \nu_{21}+\nu_{22}$.

As far as we can see, given the criteria for invariance under the prescribed symmetries and renormalizability, the potential $V[\Phi]$ so constructed is unique. 


\section{The Framon Potential Vacuum}

Our next job is to find the minima of the framon potential to identify the vacuum which, as we shall see, has some intriguing properties with interesting consequences. The problem is of course in principle entirely soluble just by differentiating the potential with respect to the various components of the framon fields, but the potential being rather complicated, it pays first to examine the problem qualitatively to see what answer one might expect. To do so, we find it convenient to express the potential in terms of the 3 -vectors $\boldsymbol{\alpha}$ and $\boldsymbol{\phi}_{a}, a=1,2,3$, thus

$$
\begin{aligned}
V[\Phi]= & -\mu_{W}|\phi|^{2}+\lambda_{W}\left(|\phi|^{2}\right)^{2} \\
& -\mu_{S} \sum_{a}\left|\phi_{a}\right|^{2}+\lambda_{S}\left(\sum_{a}\left|\boldsymbol{\phi}_{a}\right|^{2}\right)^{2}+\kappa_{S} \sum_{a, b}\left|\left(\boldsymbol{\phi}_{a}^{*} \cdot \boldsymbol{\phi}_{b}\right)\right|^{2} \\
& +\nu_{1}|\boldsymbol{\phi}|^{2} \sum_{a}\left|\boldsymbol{\phi}_{a}\right|^{2}-\nu_{2}|\boldsymbol{\phi}|^{2} \sum_{a}\left|\left(\boldsymbol{\alpha}^{*} \cdot \boldsymbol{\phi}_{a}\right)\right|^{2} .
\end{aligned}
$$

Notice that $\boldsymbol{\alpha}$ and $\boldsymbol{\phi}_{a}, a=1,2,3$ here are vectors in $\widetilde{s u}(3)$ space, not in $s u(3)$ space as were the vectors $\phi^{\tilde{a}}$ in the expression (3.8) for the kinetic energy term $\mathcal{A}_{K E}$. The signs of the coefficients $\mu, \lambda, \kappa$ and $\nu$ are all in principle arbitrary as far as invariance is concerned, but we shall choose them all to be positive for the present discussion. The chosen signs for the $\mu$ 's and $\lambda$ 's are the same as for the standard electroweak theory and are not therefore new. The signs chosen for $\nu_{1}$ and $\nu_{2}$ are not really necessary for our purpose as far as we can see, but are chosen such only for ease of presentation. This leaves then the sign chosen for $\kappa_{S}$ as the only genuinely new assumption.

To study the minimization problem of the above potential (4.1), we shall proceed in 2 steps. We shall consider first the 2 parts of the potential labelled previously as $V_{W}$ and $V_{S}$, the former depending only on the weak framon $\phi$ and the latter depending only on the strong framons $\phi_{a}$. Then secondly, we shall examine the effect of the remaining terms linking the weak and strong sectors, namely $\nu_{1}$ and $\nu_{2}$, which we shall treat as disturbances on the purely weak and purely strong potentials.

The weak potential comprising the first 2 terms in (4.1) is the same as in standard electroweak theory with minimum at $|\phi|^{2}=\mu_{W} /\left(2 \lambda_{W}\right)$ of which nothing more needs to be said. The strong potential consisting of the following 3 terms in (4.1) has more intricate features. We notice first that the $\kappa_{S}$ term is the only term which depends on the relative orientations among the vectors $\phi_{a}, a=1,2,3$, the other 2 terms $\mu_{S}$ and $\lambda_{S}$ being functions 
only of their lengths $\left|\phi_{a}\right|$. Hence one can minimize first the $\kappa_{S}$ term with respect to the orientations of the vectors, and obtain for $\kappa_{S}$ positive that the 3 vectors $\phi_{a}, a=1,2,3$ at minimum are mutually orthogonal. This means that at minimum one can omit from the $\kappa_{S}$ term all those terms with $a \neq b$ leaving:

$$
V_{S R}[\Phi]=-\mu_{S} \sum_{a}\left|\phi_{a}\right|^{2}+\lambda_{S}\left(\sum_{a}\left|\phi_{a}\right|^{2}\right)^{2}+\kappa_{S} \sum_{a}\left(\left|\phi_{a}\right|^{2}\right)^{2},
$$

depending only on the lengths $\left|\phi_{a}\right|$, and minimising it with respect to these easily gives:

$$
\left|\phi_{1}\right|^{2}=\left|\phi_{2}\right|^{2}=\left|\phi_{3}\right|^{2}=\frac{\mu_{S}}{6 \lambda_{S}+2 \kappa_{S}} .
$$

In other words, at minimum, the framon fields $\phi_{a}$ when normalized would form an orthonormal triad, exactly as what one would expect for frame vectors. A little more detail for the properties of the strong potential $V_{S}$ can be found in [1].

We turn now to the terms $\nu_{1}$ and $\nu_{2}$ linking the weak and strong sectors. The $\nu_{1}$ term affects only the squared length $|\phi|^{2}$ of the weak framon and the squared length:

$$
\zeta_{S}^{2}=\sum_{a}\left|\phi_{a}\right|^{2}
$$

of the strong, and present thus no very new features. We shall thus first focus on the influence of the $\nu_{2}$ term on the framons in the strong sector. We shall regard this effect as perturbative, seeing that the $\nu$ terms as compared with the terms in the strong potential are of the order $|\phi|^{2} /\left|\phi_{a}\right|^{2}$, which we have reason to believe is small. However, this is just a matter of convenience, for most of what we conclude would still hold even if this turns out not to be true.

We recall that, because of the $\kappa_{S}$ term in the strong potential, its minimization implies that the framons $\phi_{a}$ form an orthogonal triad all of the same length. We ask now what will happen when we minimize the $\kappa_{S}$ and $\nu_{2}$ terms together. Consider first the situation when these framons are kept still having the same length, thus allowing only their orientations to vary. For the $+\operatorname{sign}$ of the $\nu_{2}$ term that we have chosen, this term is smallest when the framons $\boldsymbol{\phi}_{a}$ are all aligned with the vector $\boldsymbol{\alpha}$, but this is opposed by the $\kappa_{S}$ term which, to attain its smallest value, would want instead the framons to be mutually orthogonal. The result of minimising the 2 terms together would thus be a compromise where the originally orthogonal triad 
is squeezed together a little towards the vector $\boldsymbol{\alpha}$ which, by the symmetry of the problem, would be placed symmetrically with respect to the triad. The amount of distortion $\delta$ to the orthogonal triad would be of the order $|\phi|^{2} /\left|\phi_{a}\right|^{2}$.

Next, consider the opposite situation when the framons are kept mutually orthogonal but allowed to change their lengths relative to one another. We recall first that the $\mu_{S}$ and $\lambda_{S}$ terms of the purely strong potential depend only on $\zeta_{S}$, not on how it is distributed among the three lengths $\left|\phi_{a}\right|$. It was the $\kappa_{S}$ term whose minimization gave the result that the 3 lengths should be equal. However, this is again opposed by the $\nu_{2}$ linkage term which, to achieve its smallest value, would prefer to have all the weight of $\zeta_{S}$ attributed to just one of the framons $\phi_{a}$ and $\boldsymbol{\alpha}$ to be aligned with it. Minimizing the 2 terms together would thus once more lead to a compromise where the minimum would be displaced from the symmetrical point favoured by the purely strong potential to a point with the lengths of the $\boldsymbol{\phi}_{a}$ 's differing from one another by amounts of the order $\zeta_{S} \delta$, and with the vector $\boldsymbol{\alpha}$ aligned with the longest.

From the conclusion in the above 2 extreme situations, it is then not difficult to visualize what will happen in the actual situation when the framons are allowed both to deviate from mutual orthogonality and to change in length relative to one another. The original orthogonal triad will be squeezed slightly by an amount of order $|\phi|^{2} /\left|\phi_{a}\right|^{2}$, while at the same time the framons will deviate in relative length from one another by amounts of the same order, with the vector $\boldsymbol{\alpha}$ snuggling up to the longest among them. Since the squeezing of the orthogonal triad has a similar effect to giving the framons different lengths in reducing the value of the potential, one can imagine a kind of trade-off between the two, or that the minimum of the potential is degenerate with respect to the amount of squeeze on the triad so long as the effect is compensated by a simultaneous change in the relative framon lengths in a prescribed manner. The degeneracy would be 2-dimensional, there being 2 angles in the triad to be squeezed or 2 relative lengths of vectors to be varied.

Next, we ask how the vector $\boldsymbol{\alpha}$ will change when the relative lengths of the strong framons vary. We note first that when the strong framons $\phi_{a}$ are strictly orthogonal to one another and have the same length, then the $\nu_{2}$ term will have no preference for the direction of $\boldsymbol{\alpha}$, the term being proportional to the sum of the squares of the direction cosines of $\boldsymbol{\alpha}$ and therefore independent of the direction of $\boldsymbol{\alpha}$. However, as soon as the framons are allowed to have different lengths, then $\boldsymbol{\alpha}$ will flop to align itself with the 
longest one for the $\nu_{2}$ term will then acquire its minimum value. But if we now allow the orthogonality also to be distorted, then $\boldsymbol{\alpha}$ can no longer align itself exactly with the longest framon for its inner products with the shorter framons will now also contribute. Hence, $\boldsymbol{\alpha}$ will end up quite closely but not exactly aligned with the longest framon. Nevertheless, for small squeezing angles, the system is close to being unstable so that even a small change in relative lengths of the framons from identity would be enough to bring about a large change in the direction of $\boldsymbol{\alpha}$. In other words, if we choose to write:

$$
\left(\left|\phi_{1}\right|,\left|\phi_{2}\right|,\left|\phi_{3}\right|\right)=\zeta_{S}(x, y, z) ; \quad x^{2}+y^{2}+z^{2}=1
$$

considering $\mathbf{r}=(x, y, z)$ itself as a unit vector so that changes in the relative lengths of $\phi_{a}$ 's can be pictured as a rotation of this vector, then our previous conclusion can be restated as saying that a small rotation of $\mathbf{r}$ will lead to a large rotation of $\boldsymbol{\alpha}$, or that the directions of $\mathbf{r}$ and $\boldsymbol{\alpha}$ are coupled such that rotations in $\mathbf{r}$ will become magnified in $\boldsymbol{\alpha}$. The smaller the value of the squeezing angle $\delta$, the closer is the system to instability and the more effective is the magnification.

All these properties of the vacuum can, of course, be derived just by minimising the framon potential (3.11). However, the algebraic complications, due mainly to the deviations of the vacuum from orthonormality, tend to obscure the basic structure of the calculation. For clarity of presentation, therefore, we shall work out in full here only a simplified version of it where there are only 2 colours (and hence only 2 fermion generations as the dual of colour) instead of the actual 3. This will contain in paraphrase already all the essential features in the actual 3-generation problem. The calculation on the vacuum and its subsequent applications for the actual 3-generation case will, in this paper, be carried only to such an approximation and extent as to satisfy ourselves that it holds no surprises beyond what is discovered from the 2-generation model. A full calculation is under way and will be reported on separately if found necessary.

By the 2-G (2 colours and 2 generations) model of the strong sector, we mean a theory with a local $s u(2)$ symmetry representing colour plus a global $\widetilde{s u}(2)$ symmetry representing generations. Such a theory can, as described at the beginning of Section 2, be formulated via (2.3) with a smaller number of framons, as with the electroweak theory. However, in order to simulate better the actual 3-G case, we shall take the framon fields here as the components of a $2 \times 2$ matrix $\Phi$, satisfying only the constraint that the determinant be real. 
Our convention, we recall is that the columns of $\Phi$ transform as doublets under local $s u(2)$ while its rows transform as anti-doublets under global $\widetilde{s u}(2)$. We can then choose to work in the (local) gauge where the first column of $\Phi$ points in the first direction and is real, the 3 degrees of freedom in $s u(2)$ being just sufficient for us to do so. The condition that the determinant of $\Phi$ be real then implies the second diagonal element to be real also. We shall refer henceforth to this as the triangular gauge, in which $\Phi$ can be parametrized as:

$$
\Phi=\left(\begin{array}{cc}
X \cos \delta & X \sin \delta e^{i \phi} \\
0 & Y
\end{array}\right) .
$$

This $\Phi$ representing the strong framon fields is coupled in the potential (3.11) to the weak sector via the vector $\boldsymbol{\alpha}$ which we parametrize in this gauge as:

$$
\boldsymbol{\alpha}=\left(\begin{array}{c}
\cos \alpha e^{-i \beta} \\
\sin \alpha e^{-i \gamma}
\end{array}\right) \text {. }
$$

We can then write the potential as:

$$
\begin{aligned}
V[\Phi]= & -\mu_{W} \zeta_{W}^{2}+\lambda_{W} \zeta_{W}^{4} \\
& -\mu_{S}\left(X^{2}+Y^{2}\right)+\lambda_{S}\left(X^{2}+Y^{2}\right)^{2}+\kappa_{S}\left(X^{4}+Y^{4}+2 X^{2} Y^{2} \sin ^{2} \delta\right) \\
& +\nu_{1} \zeta_{W}^{2}\left(X^{2}+Y^{2}\right) \\
& -\nu_{2} \zeta_{W}^{2}\left[(X \cos \delta \cos \alpha+X \sin \delta \sin \alpha \cos \theta)^{2}+X^{2} \sin ^{2} \delta \sin ^{2} \alpha \sin ^{2} \theta\right] \\
& -\nu_{2} \zeta_{W}^{2} Y^{2} \sin ^{2} \alpha,
\end{aligned}
$$

as a function of the 6 variables $X, Y, \delta, \alpha$,

$$
\zeta_{W}^{2}=|\phi|^{2}, \quad \text { and } \quad \theta=\phi+\beta-\gamma .
$$

The vacuum of the potential is to be obtained by putting the derivatives of $V$ with respect to all 6 variables to zero. First, differentiating with respect to $\theta$, we easily obtain that the minimum occurs at $\theta=0$. Differentiating with respect to the remaining 5 variables, we then obtain, for $\theta=0$ :

$$
\begin{aligned}
\zeta_{S}^{2} & =\frac{2 \mu_{S}+\nu_{2} \zeta_{W}^{2}\left[\cos ^{2}(\alpha-\delta)+\sin ^{2} \alpha\right]-2 \nu_{1} \zeta_{W}^{2}}{4 \lambda_{S}+2 \kappa_{S}\left(1+\sin ^{2} \delta\right)}, \\
\Delta & =\left(\frac{\nu_{2} \zeta_{W}^{2}}{2 \kappa_{S} \zeta^{2}}\right) \frac{\cos (2 \alpha-\delta)}{\cos \delta}, \\
\sin 2 \delta & =2\left(\frac{\nu_{2} \zeta_{W}^{2}}{2 \kappa_{S} \zeta^{2}}\right) \frac{\sin 2 \alpha}{1+\Delta}, \\
\sin 2(\alpha-\delta) & =\left(\frac{1-\Delta}{1+\Delta}\right) \sin 2 \alpha,
\end{aligned}
$$


and

$$
\zeta_{W}^{2}=\frac{2 \mu_{W}+\nu_{2} \zeta_{S}^{2}\left[(1+\Delta) \cos ^{2}(\alpha-\delta)+(1-\Delta) \sin ^{2} \alpha\right]-2 \nu_{1} \zeta_{S}^{2}}{4 \lambda_{W}},
$$

where we have written:

$$
X=\zeta_{S} x, \quad Y=\zeta_{S} y,
$$

with

$$
\Delta=x^{2}-y^{2}, \quad x^{2}+y^{2}=1 .
$$

Interestingly, these equations can be solved explicitly as follows. We notice first that the equation (4.13) is dependent on the 2 equations before it, and can thus be omitted. This leaves then only 4 equations in all for our 5 unknowns $\zeta_{S}, \zeta_{W}, \Delta, \delta, \alpha$, meaning thus that the vacuum has a 1 -dimensional degeneracy. Next, we see that the 2 equations (4.12) and (4.13) are solved by:

$$
\sin \delta=\sqrt{\frac{R^{2}-\Delta^{2}}{1-\Delta^{2}}}, \quad \sin \alpha=\sqrt{\frac{(R-\Delta)(1+R)}{2 R(1-\Delta)}},
$$

in terms of $\Delta$, or alternatively by:

$$
\Delta=\frac{R(R+\cos 2 \alpha)}{1+R \cos 2 \alpha} \quad \sin \delta=\frac{R \sin 2 \alpha}{\sqrt{1+R^{2}+2 R \cos 2 \alpha}},
$$

in terms of $\alpha$, with:

$$
R=\frac{\nu_{2} \zeta_{W}^{2}}{2 \kappa_{S} \zeta_{S}^{2}}
$$

Substitution of these results into the remaining equations then gives after some algebra:

$$
\begin{aligned}
\zeta_{S}^{2} & =\frac{\mu_{S}}{2 \lambda_{S}+2 R \kappa_{S}\left(\nu_{1} / \nu_{2}\right)+\kappa_{S}(1-R)} \\
\zeta_{W}^{2} & =\frac{2 \mu_{W}+\nu_{2} \zeta_{S}^{2}(1+R)-2 \nu_{1} \zeta_{S}^{2}}{4 \lambda_{W}} \\
R & =\frac{2 \mu_{W} \lambda_{S} \nu_{2}+\kappa_{S} \nu_{2} \mu_{W}+\nu_{2}^{2}-2 \nu_{1} \nu_{2}}{8 \lambda_{W} \mu_{S} \kappa_{S}-2 \kappa_{S} \mu_{W} \nu_{1}+\kappa_{S} \nu_{2} \mu_{W}-\nu_{2}^{2}},
\end{aligned}
$$

where we notice that $R$ is independent of $\Delta$ or $\alpha$, i.e. constant over the degenerate vacuum, and hence so are also $\zeta_{S}$ and $\zeta_{W}$ by (4.20) and (4.21). 
These results confirm the conclusions of the qualitative arguments above that both the deviations $\Delta$ and $\delta$ of the framons from orthonormality are proportional to the supposedly small parameter $R$, and that changes in $\Delta$ and/or $\delta$ of order $R$ are enough to produce changes in $\alpha$ of order unity. The solution (4.18) in terms of $\alpha$ is particularly convenient in that it can be extended to all $\alpha$ ranging from 0 to $2 \pi$, i.e. over all 4 quadrants. For $\alpha=n \pi / 2$, i.e. when the vector $\boldsymbol{\alpha}$ is pointing along any of the coordinate axes, then $\delta=0$ or that the framons are orthogonal, but are not of the same length, $\Delta= \pm R$. In between, the framons deviate from orthogonality with $\delta>0$ for $\boldsymbol{\alpha}$ pointing in the first and third quadrants, but $\delta<0$ in the second and the fourth.

The analogous problem in the actual 3-G case, though much more complicated, can be worked out along similar lines. We choose again to work in the triangular gauge where the framon field can be parametrized as:

$$
\Phi=\left(\begin{array}{ccc}
X \cos \delta_{1} & X \sin \delta_{1} \sin \gamma e^{i \chi_{3}} & X \sin \delta_{1} \cos \gamma e^{i \chi_{2}} \\
0 & Y \cos \delta_{2} & Y \sin \delta_{2} e^{i \chi_{1}} \\
0 & 0 & Z
\end{array}\right)
$$

and the vector $\boldsymbol{\alpha}$ as:

$$
\boldsymbol{\alpha}=\left(\begin{array}{l}
\cos \theta e^{-i \beta_{1}} \\
\sin \theta \sin \phi e^{-i \beta_{2}} \\
\sin \theta \cos \phi e^{-i \beta_{3}}
\end{array}\right)
$$

Substituting these into the formula (3.11) one obtains for the framon potential an expression:

$$
\begin{aligned}
V[\Phi]= & -\mu_{W} \zeta_{W}^{2}+\lambda_{W} \zeta_{W}^{4} \\
& -\mu_{S}\left(X^{2}+Y^{2}+Z^{2}\right)+\lambda_{S}\left(X^{2}+Y^{2}+Z^{2}\right)^{2} \\
& +\kappa_{S}\left\{X^{4}+Y^{4}+Z^{4}+2 X^{2} Y^{2} \sin ^{2} \delta_{1} \sin ^{2} \gamma \cos ^{2} \delta_{2}\right. \\
& +2 X^{2} Y^{2} \sin ^{2} \delta_{1} \cos ^{2} \gamma \sin ^{2} \delta_{2}+2 X^{2} Z^{2} \sin ^{2} \delta_{1} \cos ^{2} \gamma \\
& \left.+2 Y^{2} Z^{2} \sin ^{2} \delta_{2}+X^{2} Y^{2} \sin ^{2} \delta_{1} \sin 2 \gamma \sin 2 \delta_{2} \cos \left(\theta_{1}+\theta_{2}-\theta_{3}\right)\right\} \\
& +\nu_{1} \zeta_{W}^{2}\left(X^{2}+Y^{2}+Z^{2}\right) \\
& -\nu_{2} \zeta_{W}^{2}\left\{X ^ { 2 } \left(\cos ^{2} \delta_{1} \cos ^{2} \theta+\sin ^{2} \delta_{1} \sin ^{2} \gamma \sin ^{2} \theta \sin ^{2} \phi\right.\right. \\
& +\sin ^{2} \delta_{1} \cos ^{2} \gamma \sin ^{2} \theta \cos ^{2} \phi+\frac{1}{2} \sin 2 \delta_{1} \sin \gamma \sin 2 \theta \sin \phi \cos \theta_{2} \\
& \left.+\frac{1}{2} \sin 2 \delta_{1} \cos \gamma \sin _{2} \theta \cos _{\phi} \cos \theta_{3}+\frac{1}{2} \sin ^{2} \delta_{1} \sin 2 \gamma \sin ^{2} \theta \sin 2 \phi \cos \left(\theta_{2}-\theta_{3}\right)\right) \\
& +Y^{2}\left(\cos ^{2} \delta_{2} \sin ^{2} \theta \sin ^{2} \phi+\sin ^{2} \delta_{2} \sin ^{2} \theta \cos ^{2} \phi+\frac{1}{2} \sin 2 \delta_{2} \sin ^{2} \theta \sin 2 \phi \cos \theta_{1}\right) \\
& \left.+Z^{2} \sin ^{2} \theta \cos ^{2} \phi\right\}
\end{aligned}
$$


in terms of the 12 variables $X, Y, Z, \delta_{1}, \delta_{2}, \gamma, \theta, \phi, \theta_{1}, \theta_{2}, \theta_{3}$ and $\zeta_{W}$ with:

$$
\theta_{1}=-\chi_{1}-\beta_{2}+\beta_{3}, \quad \theta_{2}=-\chi_{3}-\beta_{1}+\beta_{2}, \quad \theta_{3}=-\chi_{2}-\beta_{1}+\beta_{3} .
$$

Again, the vacuum is to be given by putting to zero the derivatives of the framon potential with respect to all these 12 variables. The resulting equations are quite complicated and have been worked out so far only to first order in $\zeta_{W}^{2} / \zeta_{S}^{2}$. First, one finds from the derivatives with respect to $\theta_{i}$ that these variables should vanish at the minimum, thus giving:

$$
\chi_{1}=\beta_{3}-\beta_{2}, \quad \chi_{2}=\beta_{3}-\beta_{1}, \quad \chi_{3}=\beta_{2}-\beta_{1},
$$

and eliminating the $\theta_{i}$ 's from the system. The remaining equations give first $\phi=\gamma$, which we use to eliminate $\phi$, leaving the following 8 equations.

$$
\begin{aligned}
\zeta_{S}^{2}= & \frac{3 \mu_{S}+\nu_{2} \zeta_{W}^{2}-3 \nu_{1} \zeta_{W}^{2}}{6 \lambda_{S}+2 \kappa_{S}} \\
\Delta_{1}= & \frac{\nu_{2} \zeta_{W}^{2}}{2 \kappa_{S} \zeta_{S}^{2}}\left(\cos ^{2} \theta-\sin ^{2} \theta \sin ^{2} \gamma\right) \\
\Delta_{2}= & -\frac{\nu_{2} \zeta_{W}^{2}}{2 \kappa_{S} \zeta_{S}^{2}} \sin ^{2} \theta \cos 2 \gamma \\
\delta_{1}= & \frac{3 \nu_{2} \zeta_{W}^{2}}{4 \kappa_{S} \zeta_{S}^{2}} \sin 2 \theta \\
\delta_{2}= & \frac{3 \nu_{2} \zeta_{W}^{2}}{4 \kappa_{S} \zeta_{S}^{2}} \sin ^{2} \theta \sin 2 \gamma, \\
\delta_{1}= & \frac{3}{2} \tan _{2} \theta\left(\Delta_{1}+\Delta_{2} \cos ^{2} \gamma-\frac{1}{3} \delta_{2} \sin 2 \gamma\right), \\
\delta_{2}= & -\frac{3}{2} \Delta_{2} \tan _{2} \gamma, \\
\zeta_{W}^{2}= & \frac{1}{6 \lambda_{W}}\left\{3 \mu_{W}-3 \nu_{1} \zeta_{S}^{2}+\nu_{2} \zeta_{S}^{2}\left(1+2 \Delta_{1}-3 \Delta_{1} \sin ^{2} \theta+\Delta_{2}\right.\right. \\
& \left.\left.-3 \Delta_{2} \sin ^{2} \theta \cos ^{2} \gamma+\delta_{1} \sin 2 \theta+\delta_{2} \sin 2 \gamma \sin ^{2} \theta\right)\right\}
\end{aligned}
$$

where we have written, in analogy to the 2-G model:

$$
X=\zeta_{S} x, \quad Y=\zeta_{S} y, \quad Z=\zeta_{S} z
$$

with

$$
\Delta_{1}=x^{2}-y^{2}, \quad \Delta_{2}=y^{2}-z^{2}, \quad x^{2}+y^{2}+z^{2}=1 .
$$


Again, it can be shown that 2 of these equations, e.g. (4.33) and (4.34), are dependent on the others and can be omitted, leaving only 6 equations for 8 unknowns, meaning thus that the vacuum has a 2-dimensional degeneracy as anticipated. Further, the equations (4.29)-(4.32) imply that the quantities $\Delta_{1}, \Delta_{2}, \delta_{1}, \delta_{2}$ are all of order $\zeta_{W}^{2} / \zeta_{S}^{2}$, and that their variations to that order will give rise already to changes in $\theta$ and $\gamma$ of order unity. In other words, all the properties surmised earlier by qualitative arguments are again confirmed.

The equations (4.29)-(4.32) are solved explicitly to leading order in terms of $\Delta_{1}, \Delta_{2}$ and $R$ as follows:

$$
\begin{aligned}
\sin \theta & =\sqrt{\frac{2 R-2 \Delta_{1}-\Delta_{2}}{3 R}}, \\
\sin \gamma & =\sqrt{\frac{R-\Delta_{1}+\Delta_{2}}{2 R-2 \Delta_{1}-\Delta_{2}}}, \\
\delta_{1} & =\sqrt{\left(R+2 \Delta_{1}+\Delta_{2}\right)\left(2 R-2 \Delta_{1}-\Delta_{2}\right)}, \\
\delta_{2} & =\sqrt{\left(R-\Delta_{1}-2 \Delta_{2}\right)\left(R-\Delta_{1}+\Delta_{2}\right)},
\end{aligned}
$$

or alternatively in terms of $\theta, \gamma$ and $R$ as:

$$
\begin{aligned}
\Delta_{1} & =R\left(\cos ^{2} \theta-\sin ^{2} \theta \sin ^{2} \gamma\right), \\
\Delta_{2} & =-R \sin ^{2} \theta \cos 2 \gamma, \\
\delta_{1} & =\frac{3}{2} R \sin 2 \theta, \\
\delta_{2} & =\frac{3}{2} R \sin ^{2} \theta \sin 2 \gamma .
\end{aligned}
$$

Substitution of (4.38) into the other equations then gives $\zeta_{W}, \zeta_{S}$, and hence $R$ also, which again all turn out to be constant, i.e. independent of $\Delta_{1}$ and $\Delta_{2}$, or of $\theta$ and $\gamma$, but the explicit expressions are not of immediate interest. Similar remarks to those above for the 2 -G model on the dependence of the vacuum on the direction of $\boldsymbol{\alpha}$ apply also to the present case. As $\boldsymbol{\alpha}$ varies over the unit sphere, the framon triad gets distorted in various ways from orthonormality, but the distortions are never larger than of order $R$.

The remarkable property of the vacuum here being degenerate and depending on the vector $\boldsymbol{\alpha}$ is of quite crucial significance to our explanation later for the fermion mass hierarchy and mixing pattern, and deserves a closer examination on its origin. We recall that the potential $V_{S}$ for the strong framon sector by itself has a vacuum which is rather featureless where the strong framons just form an orthogonal triad all having the same length. 
Nevertheless, this vacuum is degenerate because of $\widetilde{s u}(3)$ invariance, meaning that one can change at will the orientation of the orthogonal triad in $\widetilde{s u}(3)$ space, although this degeneracy is not of much interest since there is no reference point to tell whether one has indeed made such a change. The vector $\boldsymbol{\alpha}$ coming from the weak sector, however, gives a reference point for the orientation and distorts at the same time the vacua from orthonormality via the $\nu_{2}$ term in the linkage part $V_{W S}$ of the framon potential, so that the vacua now look different from one another depending on the value of $\boldsymbol{\alpha}$. One can say that it is the weak sector $s u(2)$ which breaks the $\widetilde{s u}(3)$ invariance of the strong sector, in a manner similar in spirit though not in details to the breaking by $u(1)$ of the weak sector symmetry $\widetilde{s u}(2)$ in the electroweak theory [6, 1]. Nevertheless, the theory overall has still $\widetilde{s u}(3)$ invariance, and so must also the set of degenerate vacua labelled by $\boldsymbol{\alpha}$. And since $\boldsymbol{\alpha}$ varies under $\widetilde{s u}(3)$ transformation, it has to follow that the different vacua labelled by different values of $\boldsymbol{\alpha}$ are equivalent under $\widetilde{s u}(3)$ transformations.

Let us work this out explicitly, again for simplicity first in the 2-G model. Let us start in a fixed $\widetilde{s u}(2)$ gauge with $\boldsymbol{\alpha}=\boldsymbol{\alpha}_{0}$, and $\boldsymbol{\alpha}_{0}^{\dagger}=(1,0)$. This corresponds to $\alpha=0$ in the notation of (4.18) and gives the vacuum in the triangular (local $\mathrm{su}(2)$ ) gauge for this value of $\boldsymbol{\alpha}$ as:

$$
\Phi_{V A C}\left(\boldsymbol{\alpha}_{0}\right)=\left(\begin{array}{cc}
\sqrt{\frac{1+R}{2}} & 0 \\
0 & \sqrt{\frac{1-R}{2}}
\end{array}\right) .
$$

Effect now an $\widetilde{s u}(2)$ transformation, say,

$$
A=\left(\begin{array}{cc}
\cos \alpha e^{-i \beta} & -\sin \alpha e^{i \gamma} \\
\sin \alpha e^{-i \gamma} & \cos \alpha e^{i \beta}
\end{array}\right)
$$

operating on $\boldsymbol{\alpha}_{0}$ from the left to give:

$$
\boldsymbol{\alpha}=A \boldsymbol{\alpha}_{0}=\left(\begin{array}{c}
\cos \alpha e^{-i \beta} \\
\sin \alpha e^{-i \gamma}
\end{array}\right) \text {. }
$$

The value of $\Phi$ for the vacuum corresponding to this new value of $\boldsymbol{\alpha}$, but still in the same $\widetilde{s u}(2)$ and $s u(2)$ gauge, is obtained by operating with $A^{-1}$ from the right on $\Phi_{V A C}\left(\alpha_{0}\right)$ above, giving:

$$
\Phi_{V A C}(\boldsymbol{\alpha})=\Phi_{V A C}\left(\boldsymbol{\alpha}_{0}\right) A^{-1}=\left(\begin{array}{cc}
\sqrt{\frac{1+R}{2}} \cos \alpha e^{i \beta} & \sqrt{\frac{1+R}{2}} \sin \alpha e^{i \gamma} \\
-\sqrt{\frac{1-R}{2}} \sin \alpha e^{-i \gamma} & \sqrt{\frac{1-R}{2}} \cos \alpha e^{-i \beta}
\end{array}\right) .
$$


This is not in the triangular form, but can be transformed into such by an $s u(2)$ transformation (i.e. a change of the local su(2) gauge) say:

$$
\Theta=\left(\begin{array}{cc}
\cos \theta e^{-i \phi} & -\sin \theta e^{i \chi} \\
\sin \theta e^{-i \chi} & \cos \theta e^{i \phi}
\end{array}\right),
$$

operating from the left, thus:

$$
\widehat{\Phi}_{V A C}(\boldsymbol{\alpha})=\Theta \Phi_{V A C}(\boldsymbol{\alpha}) .
$$

A straightforward calculation then shows that $\widehat{\Phi}_{V A C}(\boldsymbol{\alpha})$ will be triangular if we take $\phi=\beta, \chi=\gamma$, and

$$
\sqrt{1+R} \sin \theta \cos \alpha=\sqrt{1-R} \cos \theta \sin \alpha,
$$

or

$$
\sin \theta=\sqrt{\frac{1-R}{1+R \cos 2 \alpha}} \sin \alpha .
$$

Substituting this into (4.45) gives:

$$
\widehat{\Phi}_{V A C}(\boldsymbol{\alpha})=\left(\begin{array}{cc}
\sqrt{\frac{1+R \cos 2 \alpha}{2}} & \frac{R \sin 2 \alpha}{\sqrt{2(1+R \cos 2 \alpha)}} e^{-i \beta+i \gamma} \\
0 & \sqrt{\frac{1-R^{2}}{2(1+R \cos 2 \alpha)}}
\end{array}\right) .
$$

Comparing this with (4.6) through (4.18) then shows that the two expressions are identical. This means that the various vacua labelled by different values of $\boldsymbol{\alpha}$ in (4.18) are indeed gauge equivalent as anticipated.

A similar conclusion should hold also for the actual 3-G case. To check that it is so, we work again to leading order in $R$. As in the $2-G$ model above, we work in the $\widetilde{s u}(3)$ gauge with $\boldsymbol{\alpha}_{0}^{\dagger}=(1,0,0)$ and in the $s u(3)$ gauge where the value of $\phi$ at the vacuum there is triangular, which means, according to (4.39), that it is actually diagonal:

$$
\Phi_{V A C}\left(\boldsymbol{\alpha}_{0}\right)=\left(\begin{array}{ccc}
\sqrt{\frac{1+2 R}{3}} & 0 & 0 \\
0 & \sqrt{\frac{1-R}{3}} & 0 \\
0 & 0 & \sqrt{\frac{1-R}{3}}
\end{array}\right) .
$$


A general vacuum in the same gauge is obtained by an $\widetilde{s u}(3)$ transformation $A^{-1}$ operating on $\Phi_{V A C}\left(\boldsymbol{\alpha}_{0}\right)$ from the right, where $A$ can as usual be parametrized as:

$$
\begin{aligned}
A= & \left(\begin{array}{ccc}
e^{i \alpha_{1}} & 0 & 0 \\
0 & e^{i \alpha_{2}} & 0 \\
0 & 0 & e^{-i \alpha_{1}-i \alpha_{2}}
\end{array}\right)\left(\begin{array}{ccc}
c_{3} & -s_{3} e^{-i \sigma_{3}} & 0 \\
s_{3} e^{i \sigma_{3}} & c_{3} & 0 \\
0 & 0 & 1
\end{array}\right) \\
& \times\left(\begin{array}{ccc}
c_{2} & 0 & -s_{2} e^{-i \sigma_{2}} \\
0 & 1 & 0 \\
s_{2} e^{i \sigma_{2}} & 0 & c_{2}
\end{array}\right)\left(\begin{array}{ccc}
1 & 0 & 0 \\
0 & c_{1} & -s_{1} e^{-i \sigma_{1}} \\
0 & s_{1} e^{i \sigma_{1}} & c_{1}
\end{array}\right),
\end{aligned}
$$

where we have abbreviated sines and cosines of various angles to $s_{i}, c_{i}$. Although the 3-G vacuum is labelled by these $A$ and not just by the vector $\boldsymbol{\alpha}$ as in the 2-G case, for the present calculation we restrict ourselves only to those $A$ 's with $s_{1}=0$, i.e. no rotation in the 23 -plane. This is sufficient to take $\boldsymbol{\alpha}_{0}$ to any $\boldsymbol{\alpha}$, say (4.24). Following then the notation there for the phases, we have for $A$ the expression:

$$
A=\left(\begin{array}{ccc}
c_{2} c_{3} e^{-i \beta_{1}} & -s_{3} e^{-i\left(\beta_{1}+\sigma_{3}\right)} & -c_{3} s_{2} e^{i\left(\beta_{3}+\beta_{2}+\sigma_{3}\right)} \\
c_{2} s_{3} e^{-i \beta_{2}} & c_{3} e^{-i\left(\beta_{2}+\sigma_{3}\right)} & -s_{2} s_{3} e^{i\left(\beta_{1}+\beta_{3}+\sigma_{3}\right)} \\
s_{2} e^{-i \beta_{3}} & 0 & c_{2} e^{i\left(\beta_{1}+\beta_{2}+\sigma_{3}\right)}
\end{array}\right) .
$$

This gives for the vacuum at $\boldsymbol{\alpha}$ :

$$
\begin{aligned}
& \Phi_{V A C}(\boldsymbol{\alpha})=\Phi_{V A C}\left(\boldsymbol{\alpha}_{0}\right) A^{-1}= \\
& \left(\begin{array}{lll}
\sqrt{\frac{1+2 R}{3}} c_{2} c_{3} e^{i \beta_{1}} & \sqrt{\frac{1+2 R}{3}} c_{2} s_{3} e^{i \beta_{2}} & \sqrt{\frac{1+2 R}{3}} s_{2} e^{i \beta_{3}} \\
-\sqrt{\frac{1-R}{3}} s_{3} e^{i\left(\beta_{1}+\sigma_{3}\right)} & \sqrt{\frac{1-R}{3}} c_{3} e^{i\left(\beta_{2}+\sigma_{3}\right)} & 0 \\
-\sqrt{\frac{1-R}{3}} s_{2} c_{3} e^{-i\left(\beta_{2}+\beta_{3}+\sigma_{3}\right)} & -\sqrt{\frac{1-R}{3}} s_{2} s_{3} e^{-i\left(\beta_{1}+\beta_{3}+\sigma_{3}\right)} & \sqrt{\frac{1-R}{3}} c_{2} e^{-i\left(\beta_{1}+\beta_{2}+\sigma_{3}\right)}
\end{array}\right)
\end{aligned}
$$

$\Phi_{V A C}(\boldsymbol{\alpha})$ can be returned to the triangular form by applying from the left an $s u(3)$ transformation $\Theta$ of the same form as $A$ in (4.51) only with $s_{i}, c_{i}$ replaced by $s_{i}^{\prime}, c_{i}^{\prime}$, where

$$
\sqrt{\frac{1+2 R}{3}} c_{2} s_{2}^{\prime}=\sqrt{\frac{1-R}{3}} s_{2} c_{2}^{\prime},
$$


and

$$
s_{2} c_{3} s_{3}^{\prime}=s_{2}^{\prime} c_{3}^{\prime} s_{3}
$$

A straightforward, though somewhat lengthy, calculation via (4.53), (4.54) and (4.39) then shows that the resulting triangular form is indeed identical to (4.23). This means that one has shown that also for the $3-G$ case the vacua for different values of $\boldsymbol{\alpha}$ are gauge equivalent to one another, although here so far only to leading order in $R$

We recall that all these intriguing properties of the vacuum arise from the special form of the framon potential which is itself a consequence of the double invariance under both the local $u(1) \times s u(2) \times s u(3)$ and the global $\tilde{u}(1) \times \widetilde{s u}(2) \times \widetilde{s u}(3)$ symmetries basic to the whole framon idea. We note in particular the delicate interplay between the electroweak and strong sectors via the linkage terms implied by the invariance, which distort the strong vacuum from its symmetry position, and couple at the same time the distorted vacua to the vector $\boldsymbol{\alpha}$ coming from the weak sector, so that if the vacuum moves, it will bring the vector $\boldsymbol{\alpha}$ with it. We see, therefore, that the chain of logic in arriving at the above results is quite intricate and tightly knit.

\section{Confinement and the Boson Spectrum}

The framon potential as given, for example, in (4.1), with negative $|\boldsymbol{\phi}|^{2}$ terms is of a form usually interpreted as typical of spontaneously broken gauge symmetries. But, as pointed out as long ago as 1978 by 't Hooft [2] and by Banks and Rabinovici [3], and again as repeatedly emphasized more recently by 't Hooft [6], the electroweak theory which has such a potential can equally be interpreted as a confining theory where the gauge symmetry $s u(2)$ remains exact, what is broken being just (in our language) the global $\widetilde{s u}(2)$ symmetry "dual" to it. And the 2 pictures, i.e. whether confinement or spontaneously broken symmetry, are mathematically equivalent in present applications of the electroweak theory, although in the long run at ultra high energies, they may diverge in physical content. For a discussion of the confinement picture in the context of the framon idea we are here exploring, see for example [1].

That being the case, we shall adopt in the present treatment, as our assumption (B), exclusively the confinement picture which we find both practically more convenient and conceptually more appealing, although much of what we do here, though perhaps not all, could equally be performed in the symmetry breaking picture. This means that we shall consider both the 
$s u(2)$ and the $s u(3)$ local gauge symmetries as confining, with only the difference that the confinement in $s u(2)$ is much deeper than in $s u(3)$. This last is a necessary assumption for the confinement picture to hold as a realistic description of nature since the confinement by colour $s u(3)$ is already revealed to us by present experiment in the sense that we have already for decades been probing into hadrons as compound states of colour confinement revealing their structure in terms of their constituents. On the other hand, the equivalent compound states in $s u(2)$ confinement, such as leptons and quarks, still appear to us as point-like in all experiments so far performed. It was thus assumed by 't Hooft and implicitly also by Banks and Rabinivici that confinement in $s u(2)$ is considerably deeper than with colour, so that only in future deep inelastic experiments with ultra high energies not yet available to us will the structure of quarks and leptons be revealed. We accept this assumption, for the moment without further question, although we hope to return to it with respect to a nonabelian version of electromagnetic duality at a later stage for reasons to be explained in the concluding section.

In the confinement picture then, let us ask what is the boson spectrum for the theory that has been constructed. To answer this question, we have first to make clear what exactly this question means. The $s u(2)$ and $s u(3)$ gauge symmetries being both confined, it follows that only states which belong to the singlet representation in both will be observable as propagating particles in free space. However, while performing a deep inelastic scattering experiment of an electron on a proton, for example, we are already probing inside the proton where coloured objects such as quarks and gluons can propagate freely, and only $s u(2)$ remains confined. Let us call this the standard model scenario, for this is indeed what the Standard Model, as it now stands, represents in terms of the scheme under consideration. In the standard model scenario then, the boson spectrum would mean a list of all su(2) singlet boson states, whether colour neutral or otherwise, which can be formed as bound or compound states from the fundamental fields by $s u(2)$ confinement. Given the assumption that $s u(2)$ confinement is much deeper than colour $s u(3)$ confinement, these states will appear as quasi-elementary under all present experimental conditions. On the other hand, one can consider also what one can call the soft hadron scenario where experimental conditions are such that one never probes inside hadrons and sees all hadrons as quasi-elementary objects as one did some decades ago. Then the boson spectrum would mean a list of the bound or compound states which can be formed by confining together, via either $s u(2)$ or $s u(3)$ confinement or both, the fundamental gauge boson and framon fields so far considered and eventually also the fundamen- 
tal fermion fields yet to be introduced.

Start then with the standard model scenario where only the electroweak $s u(2)$ but not colour su(3) confines. Now of the fundamental boson fields introduced in section 2 , only the weak gauge field $B_{\mu}$ and framon field $\phi$ carry (local) $s u(2)$ indices and have to be confined; the others, i.e. the colour gauge fields $C_{\mu}$ and strong framon field $\phi_{a}$, being already (local) $s u(2)$ singlets, can exist as freely propagating particles inside hadrons. What is then the boson spectrum in such a situation?

We notice that, apart from the $\nu_{1}, \nu_{2}$ terms in the framon potential linking the weak and strong framon sectors, the part of the action involving the weak gauge and framon fields $B_{\mu}$ and $\phi$ in the present framework is identical to that of the standard electroweak theory. Hence, an analysis similar to that in [2, 3, 1] for the pure electroweak theory is expected to give for the boson spectrum in $s u(2)$ confinement essentially the same result. Explicitly, let us choose to work in the gauge when the framon field $\phi$ points in the up direction and is real. In this gauge, the part of the action involving only the weak fields will reduce to the standard electroweak action in the symmetry breaking picture evaluated in the unitary gauge when the vacuum expectation value for $\phi$ points in the up direction and is real, with only the difference that the $s u(2)$ gauge field $B_{\mu}$ is everywhere replaced by its gauge transform:

$$
\tilde{B}_{\mu}=\frac{i}{g_{2}} \Omega^{\dagger}\left(\partial_{\mu}-i g_{2} B_{\mu}\right) \Omega
$$

where $\Omega$ is the transformation we have used above to fix the gauge. Notice that $\Omega^{\dagger}$ being the transformation which rotates the local su(2) frame to align it with the global $\widetilde{s u}(2)$ frame, $\Omega$ transforms from the left under $s u(2)$ but from the right under $\widetilde{s u}(2)$, so that $\tilde{B}_{\mu}$ in (5.1), with all $s u(2)$ indices saturated, is an $s u(2)$ singlet. Indeed, to leading order in the fluctuations $H$ of $|\phi|=F+H$ about its vacuum value $F=\sqrt{\mu_{W} / 2 \lambda_{W}}$ at the minimum of the potential $V_{W}$, we can write:

$$
\operatorname{Tr}\left(\Phi^{\dagger}\left(\partial_{\mu}-i g_{2} B_{\mu}\right) \Phi\right) \approx i g_{2} \tilde{B}_{\mu}+\cdots,
$$

where $\Phi$ is the $2 \times 6$ matrix representing the "weak" framon field $\phi_{r}^{\tilde{r} \tilde{a}}$, and the trace $\operatorname{Tr}$ is taken over only the $\widetilde{s u}(3)$ indices. We see thus that the massive vector boson $\tilde{B}_{\mu}$, which in the conventional treatment is considered to be the gauge boson having acquired a mass via symmetry breaking, is now to be regarded as a $p$-wave bound state of the "weak" framon with its own conjugate. Similarly, it can be seen from:

$$
\operatorname{tr}\left(\operatorname{Tr}\left(\Phi^{\dagger} \Phi\right)\right) \approx F^{2}+2 F H+\cdots
$$


where the 2 traces are taken over $\tilde{r}$ and $\tilde{a}$ indices, that the standard Higgs boson $H$ is now to be regarded as the $s$-wave bound state of the framonanti-framon pair. But since the action is the same as in the pure electroweak theory in the symmetry breaking picture except for these reinterpretations, we recover the same mass spectrum with the familiar mixing between $\gamma$ and $Z$. Indeed, apart from the trace over $\tilde{a}$ indices, these arguments are very similar to those in the pure electroweak theory, some more details of which can be found e.g. in [1].

There is one difference, however, with the conventional electroweak theory in that because the "weak" framons here carry a $\tilde{u}(1)$ charge, so also will their bound states. This can easily be worked out for the various states just by summing the charges of the constituents and are listed below together with the $u(1)$ charge as $[q, \tilde{q}]$ :

$$
\begin{aligned}
H & \sim \operatorname{Tr}\left(\Phi^{\dagger} \Phi\right), & & {[0,0] ; } \\
W_{\mu}^{+} & \sim \phi^{(-) \dagger}\left(A_{\mu}^{i} \tau_{i}\right) \phi^{(+)}, & & {[1,-1] ; } \\
W_{\mu}^{-} & \sim \phi^{(+) \dagger}\left(A_{\mu}^{i} \tau_{i}\right) \phi^{(-)}, & & {[-1,1] ; } \\
\gamma-Z & \sim \phi^{( \pm) \dagger}\left(A_{\mu}^{i} \tau_{i}\right) \phi^{( \pm)}, & & {[0,0] . }
\end{aligned}
$$

The question of what this $\tilde{u}(1)$ charge $\tilde{q}$ signifies will be postponed until fermions are introduced in the next section

Apart from this small but, as we shall see, important, difference of a new conserved quantum number $\tilde{q}$, the result obtained above in the standard model scenario for the purely weak sector is otherwise seen to be identical to the conventional electroweak theory. Together with the colour gauge boson $C_{\mu}$, i.e. the gluon, which in the standard model scenario remains massless, these make up exactly the boson content of the Standard Model. Hence, we conclude that in the standard model scenario, the present scheme differs in the boson content from the Standard Model only in the strong framons $\phi_{a}$. Thus, so long as these last do not spoil the usual comparison of QCD with experiment as, for example, in the running of the coupling $\alpha_{s}$, which we have hope to show later, for the reason mentioned in the concluding section, to be indeed the case, we can claim consistency. The $\nu_{1}, \nu_{2}$ terms in the framon potential so far neglected will mix the Higgs boson $H$ with hadron states in a manner to be made explicit later, which will change somewhat the properties of $H$ and its mass but not the boson content in the standard model scenario.

The above conclusion on the boson content in the standard model scenario is obtained by essentially just a paraphrase in our more complex framework of 't Hooft's and Banks and Rabinovici's original arguments which took 
account only of the lowest bound states formed from a framon-antiframon pair via $s u(2)$ confinement. However, if we were to take seriously the confinement picture not just as a convenience, or alternative to the conventional symmetry-breaking picture for the electroweak theory, but as actually physical, then it would be difficult to exclude the possibility of there being other boson states formed by su(2) confinement, such as radial and orbital excitations of the ground states, familiar to hadron spectroscopists in the parallel scenario of colour su(3) confinement. We have then to envisage a situation where the actual boson spectrum is considerably richer than that seen at present, where the additional states, being presumably much heavier than the ground states listed, are not noticeable at present, but will be revealed later when higher experimental energies become available. Such a situation will obtain not just in our special framework, but in any model where the confinement picture for the electroweak theory is taken as physical [10, 11, 12, 13], and has been analysed in this context extensively and in detail already in the early paper by Claudson, Farhi and Jaffe [12]. They not only listed the many additional states possible, some with quite exotic features, but also examined the effects these can have on the physics accessible to present energies, for example those on the electromagnetic form factors of quarks and leptons. Apart from other results, their analysis on the latter effects confirms the intuition one had that, provided the additional states are sufficiently heavy, they would not disturb the correspondence between the confinement and symmetry-breaking pictures proposed. However, this still leaves the question why the ground vector boson states $W$ and $Z-\gamma$ are so much lighter than their radial and orbital excitations, in seeming contrast to what is seen in the hadron spectrum obtained in the parallel case of colour su(3) confinement. At one's present stage of understanding of $s u(2)$ confinement, unfortunately, one is still in no better position to answer this question than in the days of [12. We note only at the speculative level an interesting possibility, to be discussed in the concluding section of this paper, which is connected to a nonabelian generalization of electromagnetic duality and to the question mentioned above of why su(2) confinement is supposedly so much deeper than colour confinement. Such questions, however, of whether the confinement picture can indeed be taken physical, and if so, what new effects or phenomena it will imply at higher energies than that presently available, can be postponed as they are not of immediate concern for our present limited purpose here of just deducing from the constructed framework some otherwise mystifying properties of the Standard Model. For this purpose, the ground state boson spectrum listed above for the standard 
model scenario is already sufficient.

Next, what about the soft hadron scenario when colour is also confined? In that case, neither the gluon $C_{\mu}$ nor the framon $\phi_{a}$ will remain free but have to be confined with one another or with coloured fermions into colour singlets to give a host of new states to the hadron spectrum. The details of this is not of immediate interest in the present context of attempting to construct a protogenic standard model. The hadron spectrum is already so complicated in the Standard Model and so little understood that some more states will not be easily detected or missed, especially if they are heavy and unstable as these new modes promise to be. In any case, we are certainly not yet at the stage to worry about such details. However, for reasons to be made clear later related to the linkage between the weak and strong sectors via the $\nu_{1}, \nu_{2}$ terms, we have particular interest in the $s$-wave hadron bound states via colour confinement of $\boldsymbol{\phi}_{a}^{\dagger} \phi_{a}$, namely the equivalents in the strong sector of the Higgs field $H$ in the electroweak sector, for which we wish now to work out the spectrum.

For the strong framon potential $V_{S}$ by itself, this was done already in [1]. We wish now, however, to take account also of the linkage $\nu_{1}, \nu_{2}$ terms which distort the strong vacuum from orthonormality and make the calculation considerably more complicated. Nevertheless, the tactics developed there can still be applied with modifications. To do so, we choose again to work in the triangular gauge, where we recall that the strong framon field $\phi_{a}$ or $\Phi$ at vacuum is parametrized as in (4.23). We are now to consider small fluctuations about the vacuum, which we represent as:

$\Phi=\left(\begin{array}{ccc}X \cos \delta_{1}+h_{1} & \left(X \sin \delta_{1} \sin \gamma+\eta_{3}\right) e^{i \chi_{3}} & \left(X \sin \delta_{1} \cos \gamma+\eta_{2}\right) e^{i \chi_{2}} \\ 0 & Y \cos \delta_{2}+h_{2} & \left(Y \sin \delta_{2}+\eta_{1}\right) e^{i \chi_{1}} \\ 0 & 0 & Z+h_{3}\end{array}\right)$

where $h_{1}, h_{2}, h_{3}$ are real and $\eta_{1}, \eta_{2}, \eta_{3}$ complex. These fluctuations give altogether 9 degrees of freedom, representing the 9 real (strong) Higgs fields formed as bound states of $\Phi^{\dagger} \Phi$. To readers more familiar with the symmetry breaking picture, it may help to add that the 8 zero modes appearing in that picture which are to be eaten up by the colour gauge bosons are here already taken out of consideration by fixing to the triangular gauge.

The fluctuations $h_{i}$ and $\eta_{i}$ span the Higgs modes but are as yet neither mass eigenstates nor do they form an orthonormal basis. Again to avoid complicated details clouding the logic, we shall work these out first in the 
2-G model, for which the parallel for (5.5) is:

$$
\Phi=\left(\begin{array}{cc}
X \cos \delta+h_{1} & (X \sin \delta+\eta) e^{i \phi} \\
0 & Y+h_{2}
\end{array}\right)
$$

First, we need to be explicit in the unitary transformation taking the original frame in which the vacuum value of $\Phi$ is triangular and the fluctuations appear as:

$$
\Phi=\left(\begin{array}{cc}
X \cos \delta+\delta \phi_{1}^{\tilde{1}} & \left(X \sin \delta+\delta \phi_{1}^{\tilde{2}}\right) e^{i \phi} \\
\delta \phi_{2}^{\tilde{1}} & Y+\delta \phi_{2}^{\tilde{2}}
\end{array}\right),
$$

to the frame where $\Phi$ itself including fluctuations is of the triangular form (5.6). This is readily seen to first order in the fluctuations to be:

$$
\Omega_{D F}=\left(\begin{array}{cc}
1-\frac{i}{X \cos \delta} \delta \phi_{1 I}^{\tilde{1}} & \frac{1}{X \cos \delta}\left(\delta \phi_{2}^{\tilde{1}}\right)^{*} e^{i \phi} \\
-\frac{1}{X \cos \delta} \delta \phi_{2}^{\tilde{1}} e^{-i \phi} & 1+\frac{i}{X \cos \delta} \delta \phi_{1 I}^{\tilde{1}},
\end{array}\right),
$$

where the subscript $I$ denotes the imaginary part. Multiplying $\Omega_{D F}$ to (5.7) and comparing with (5.6) then gives:

$$
\begin{aligned}
h_{1} & =\delta \phi_{1 R}^{\tilde{1}} \\
h_{2} & =\delta \phi_{2 R}^{\tilde{2}}-(\tan \delta) \delta \phi_{2 R}^{\tilde{1}} \\
\eta_{R} & =\delta \phi_{1 R}^{\tilde{2}}+\frac{y}{x \cos \delta} \delta \phi_{2 R}^{\tilde{1}} \\
\eta_{I} & =\delta \phi_{1 I}^{\tilde{2}}-\frac{y}{x \cos \delta} \delta \phi_{2 I}^{\tilde{1}}-(\tan \delta) \delta \phi_{1 I}^{\tilde{1}},
\end{aligned}
$$

where $R$ denotes the real part. We can consider then the coefficients of $\delta \phi_{a R}^{\tilde{a}}$ and $\delta \phi_{a I}^{\tilde{a}}$ as an 8-vector. The vectors representing $h_{1}, h_{2}, \eta_{R}, \eta_{I}$ above are not all normalized nor all mutually orthogonal as 8-vectors. But we can construct easily from these via the standard Gram-Schmidt procedure an orthonormal basis for the 4-dimensional subspace they span, say $H_{1}, H_{2}, H_{R}, H_{I}$, which are conveniently represented as $2 \times 2$ complex matrices, giving respectively:

$$
\begin{aligned}
\widehat{V}_{1} & =\left(\begin{array}{ll}
1 & 0 \\
0 & 0
\end{array}\right) \\
\widehat{V}_{2} & =\sqrt{x^{2} \cos ^{2} \delta+y^{2}}\left(\begin{array}{cc}
0 & \frac{x y \sin \delta}{x^{2} \cos ^{2} \delta+y^{2}} e^{i \phi} \\
-\frac{x^{2} \sin \delta \cos \delta}{x^{2} \cos ^{2} \delta+y^{2}} e^{-i \phi} & 1
\end{array}\right)
\end{aligned}
$$




$$
\begin{aligned}
\widehat{V}_{R} & =\frac{1}{\sqrt{x^{2} \cos ^{2} \delta+y^{2}}}\left(\begin{array}{cc}
0 & x \cos \delta e^{i \phi} \\
y e^{-i \phi} & 0
\end{array}\right) \\
\widehat{V}_{I} & =i\left(\begin{array}{cc}
-x \sin \delta & x \cos \delta e^{i \phi} \\
-y e^{-i \phi} & 0
\end{array}\right) .
\end{aligned}
$$

The mass matrix of these Higgs states $H_{K}$ can be worked out by substituting (5.6) into the expression for the framon potential and expanding to second order in the fluctuations $h_{1}, h_{2}, \eta_{R}, \eta_{I}$ and $H$ (the fluctuation of the weak framon $\phi$ about its vacuum value), obtaining a quadratic form in these. One then re-expresses this form in terms of the orthonormal basis $H_{1}, H_{2}, H_{R}, H_{I}$ listed above. The coefficients of the various terms then give the Higgs mass matrix, which is a little involved. Since we shall not need this in detail, we shall give the answer only to first order in our supposedly small parameter $\zeta_{W} / \zeta_{S}$ :

$$
M_{H}=\left(\begin{array}{ccccc}
0 & \zeta_{W} \zeta_{S}\left(2 \nu_{1}-\nu_{2}\right) & \zeta_{W} \zeta_{S} \nu_{2} \cos 2 \alpha & -\zeta_{W} \zeta_{S} \nu_{2} \sin 2 \alpha & 0 \\
* & 2\left(2 \lambda_{S}+\kappa_{S}\right) \zeta_{S}^{2} & -2\left(\lambda_{S}+\kappa_{S}\right) \zeta_{S}^{2} \Delta & 2\left(\lambda_{S}+\kappa_{S}\right) \zeta_{S}^{2} \delta & 0 \\
* & * & 2 \kappa_{S} \zeta_{S}^{2} & 0 & 0 \\
* & * & * & 2 \kappa_{S} \zeta_{S}^{2} & 0 \\
* & * & * & * & 2 \kappa_{S} \zeta_{S}^{2}
\end{array}\right)
$$

where the rows and columns are labelled, in order, by $H, H_{+}, H_{-}, H_{R}, H_{I}$ with $H_{ \pm}=(1 / \sqrt{2})\left( \pm H_{1}+H_{2}\right)$. We note only that the mass eigenstates will mix the "real" states $H, H_{+}, H_{-}, H_{R}$ but not the "imaginary" state $H_{I}$, the mixing being of order $\zeta_{W} / \zeta_{S}$, or lower, so that it can in principle be worked out by perturbation theory to that order. For what follows, however, we shall not need the explicit form of the mass eigenstates.

The calculation for the actual 3-G case starting from (5.5) follows along the same lines but the algebra is more complicated. We give here the transformation $\Omega_{D F}$ to first order in the fluctuations $\delta \phi_{a}^{\tilde{a}}$ :

$$
\Omega_{D F}=\left(\begin{array}{lll}
\Omega_{11} & \Omega_{12} & \Omega_{13} \\
\Omega_{21} & \Omega_{22} & \Omega_{23} \\
\Omega_{31} & \Omega_{32} & \Omega_{33}
\end{array}\right),
$$


where

$$
\begin{aligned}
\Omega_{11}= & 1-\frac{i}{X \cos \delta_{1}}\left(\delta \phi_{1}^{\tilde{1}}\right)_{I} \\
\Omega_{12}= & \frac{\left(\delta \phi_{2}^{\tilde{1}}\right)^{*} e^{i \chi_{3}}}{X \cos \delta_{1}} \\
\Omega_{13}= & \frac{\left(\delta \phi_{3}^{\tilde{1}}\right)^{*} e^{i \chi_{2}}}{X \cos \delta_{1}} \\
\Omega_{21}= & -\frac{\delta \phi_{2}^{\tilde{1}} e^{-i \chi_{3}}}{X \cos \delta_{1}} \\
\Omega_{22}= & 1-\frac{i\left(\delta \phi_{2}^{2}\right)_{I}}{Y \cos \delta_{2}}+\frac{i \tan \delta_{1} \sin \gamma\left(\delta \phi_{2}^{\tilde{1}}\right)_{I}}{Y \cos \delta_{2}} \\
\Omega_{23}= & \frac{\left(\delta \phi_{3}^{\tilde{2}}\right)^{*} e^{i \chi_{1}}}{Y \cos \delta_{2}}-\frac{\tan \delta_{1} \sin \gamma\left(\delta \phi_{3}^{\tilde{1}}\right)^{*} e^{i \chi_{1}}}{Y \cos \delta_{2}} \\
\Omega_{31}= & -\frac{\delta \phi_{3}^{\tilde{1}} e^{-i \chi_{2}}}{X \cos \delta_{1}} \\
\Omega_{32}= & -\frac{\delta \phi_{3}^{2} e^{-i \chi_{1}}}{Y \cos \delta_{2}}+\frac{\tan \delta_{1} \sin \gamma \delta \phi_{3}^{\tilde{1}} e^{-i \chi_{1}}}{Y \cos \delta_{2}} \\
\Omega_{33}= & 1-\frac{i\left(\delta \phi_{3}^{\tilde{3}}\right)_{I}}{Z}+\frac{i \tan \delta_{1} \cos \gamma\left(\delta \phi_{3}^{\tilde{1}}\right)_{I}}{Z}+\frac{i \tan \delta_{2}\left(\delta \phi_{3}^{\tilde{2}}\right)_{I}}{Z} \\
& -\frac{i \tan \delta_{1} \tan \delta_{2} \sin \gamma\left(\delta \phi_{3}^{\tilde{1}}\right)_{I}}{Z} \\
Z &
\end{aligned}
$$

and to first order in $R$ the 9 orthonormal Higgs states in the analogous matrix notation of (5.10) which will be of use later:

$$
\begin{aligned}
\widehat{V}_{1} & =\left(\begin{array}{lll}
1 & 0 & 0 \\
0 & 0 & 0 \\
0 & 0 & 0
\end{array}\right) \\
\widehat{V}_{2} & =\left(\begin{array}{ccc}
0 & \frac{\delta_{1} x y \sin \gamma}{x^{2}+y^{2}} e^{i \chi_{3}} & 0 \\
-\frac{\delta_{1} x^{2} \sin \gamma}{x^{2}+y^{2}} e^{-i \chi_{3}} & 1 & 0 \\
0 & 0 & 0
\end{array}\right)
\end{aligned}
$$




$$
\begin{aligned}
& \widehat{V}_{3}=\left(\begin{array}{ccc}
0 & 0 & \frac{\delta_{1} x z \cos \gamma}{x^{2}+z^{2}} e^{i \chi_{2}} \\
0 & 0 & \frac{\delta_{2} y z}{y^{2}+z^{2}} e^{i \chi_{1}} \\
-\frac{\delta_{1} x^{2} \cos \gamma}{x^{2}+z^{2}} e^{-i \chi_{2}} & -\frac{\delta_{2} y^{2}}{y^{2}+z^{2}} e^{-i \chi_{1}} & 1
\end{array}\right) \\
& \widehat{W}_{1}^{R}=\frac{1}{\sqrt{y^{2}+z^{2}}}\left(\begin{array}{ccc}
0 & \frac{\delta_{1} x y^{2} \cos \gamma}{x^{2}+y^{2}} e^{i \chi_{3}} & \frac{\delta_{1} x z^{2} \sin \gamma}{x^{2}+z^{2}} e^{i \chi_{2}} \\
-\frac{\delta_{1} x^{2} y \cos \gamma}{x^{2}+y^{2}} e^{-i \chi_{3}} & 0 & y e^{i \chi_{1}} \\
-\frac{\delta_{1} x^{2} z \sin \gamma}{x^{2}+z^{2}} e^{-i \chi_{2}} & z e^{-i \chi_{1}} & 0
\end{array}\right) \\
& \widehat{W}_{2}^{R}=\frac{1}{\sqrt{x^{2}+z^{2}}}\left(\begin{array}{ccc}
0 & -\frac{\delta_{2} x y^{2}}{x^{2}+y^{2}} e^{i \chi_{3}} & x e^{i \chi_{2}} \\
\frac{\delta_{2} x^{2} y}{x^{2}+y^{2}} e^{-i \chi_{3}} & 0 & 0 \\
z e^{-i \chi_{2}} & 0 & 0
\end{array}\right) \\
& \widehat{W}_{3}^{R}=\frac{1}{\sqrt{x^{2}+y^{2}}}\left(\begin{array}{ccc}
0 & x e^{i \chi_{3}} & 0 \\
y e^{-i \chi_{3}} & 0 & 0 \\
0 & 0 & 0
\end{array}\right) \\
& \widehat{W}_{1}^{I}=\frac{i}{\sqrt{y^{2}+z^{2}}}\left(\begin{array}{ccc}
0 & -\frac{\delta_{1} x y^{2} \cos \gamma}{x^{2}+y^{2}} e^{i \chi_{3}} & \frac{\delta_{1} x z^{2} \sin \gamma}{x^{2}+z^{2}} e^{i \chi_{2}} \\
-\frac{\delta_{1} x^{2} y \cos \gamma}{x^{2}+y^{2}} e^{-i \chi_{3}} & -\delta_{2} y & y e^{i \chi_{1}} \\
\frac{\delta_{1} z x^{2} \sin \gamma}{x^{2}+z^{2}} e^{-i \chi_{2}} & -z e^{-i \chi_{1}} & 0
\end{array}\right) \\
& \widehat{W}_{2}^{I}=\frac{i}{\sqrt{x^{2}+z^{2}}}\left(\begin{array}{ccc}
-x \delta_{1} \cos \gamma & -\frac{\delta_{2} x y^{2}}{x^{2}+y^{2}} e^{i \chi_{3}} & x e^{i \chi_{2}} \\
-\frac{\delta_{2} x^{2} y}{x^{2}+y^{2}} e^{-i \chi_{3}} & 0 & 0 \\
-z e^{-i \chi_{2}} & 0 & 0
\end{array}\right) \\
& \widehat{W}_{3}^{I}=\frac{i}{\sqrt{x^{2}+y^{2}}}\left(\begin{array}{ccc}
-x \delta_{1} \sin \gamma & x e^{i \chi_{3}} & 0 \\
-y e^{-i \chi_{3}} & 0 & 0 \\
0 & 0 & 0
\end{array}\right) \text {. }
\end{aligned}
$$




\section{The Fermion Sector}

Extending next our consideration to the fermion sector, we have first to specify what we are to take as our fundamental fermion fields, which are, of course, to be given as representations of the gauge symmetries of the theory, namely $u(1) \times s u(2) \times s u(3)$. Not having ascribed in our framework any geometrical significance to fermion fields as we did to the vector and scalar fields in the boson sector, we are thrown back as usual to arguments of simplicity. On this guideline then, let us take as fundamental fermion fields the following:

$$
\psi=\psi(1,1) ; \quad \psi_{r}=\psi(2,1) ; \quad \psi_{a}=\psi(1,3) ; \quad \psi_{r a}=\psi(2,3),
$$

where inside the brackets, the first argument denotes the dimension of the representation of the $s u(2)$ symmetry, the second argument that of the $s u(3)$ symmetry. These are the simplest possibilities involving as they do only the singlet and fundamental representations of either $s u(2)$ or $s u(3)$.

We have yet to specify the $u(1)$ charges $q$ of the $\psi$ 's in (6.1), if any. Recall now the discussion in section 2 on the choice of the gauge group, which concluded with the choice of the group $U(1,2,3)$ defined there. The admissible $u(1)$ charges for the fermions (6.1) which are of necessity representations of the group $U(1,2,3)$ can then be read off from (2.9). Let us concentrate first on the $s u(2)$ doublets $\psi(2,1)$ and $\psi(2,3)$ and restrict ourselves as usual to those with the smallest values of $|q|$, obtaining:

$$
\begin{aligned}
\psi_{r}^{( \pm 1 / 2)} & =\psi^{( \pm 1 / 2)}(2,1) ; & & q= \pm \frac{1}{2}, \\
\psi_{r a}^{(1 / 6)} & =\psi^{(1 / 6)}(2,3) ; & & q=\frac{1}{6},
\end{aligned}
$$

where when necessary, the $u(1)$ charge $q$ will be carried as a bracketed superscript as shown.

Notice that the fundamental fermion fields, like the gauge boson fields, do not, and have no reason to, carry any indices referring to the global symmetries $\tilde{u}(1) \times \widetilde{s u}(2) \times \widetilde{s u}(3)$. These have to do only with the global reference frames and should thus affect only the framons.

In the confinement picture in which we work, only singlets of the nonabelian local symmetries can exist as freely propagating particles. Let us ask then what fermion spectrum we are to expect. Again, the answer depends on whether we are in the standard model or soft hadron scenario. Our primary concern is the standard model scenario where experimental conditions 
are at present, namely where colour $s u(3)$ confinement has already been revealed, but $s u(2)$ confinement is not, so that only $s u(2)$ singlets are observed as freely propagating and appear to us as elementary objects. In that case, only $\psi(1,1)$ and $\psi(1,3)$ in (6.1) can appear free but $\psi(2,1)$ and $\psi(2,3)$ have to be confined.

As in the pure electroweak theory treated by 't Hooft [2] and Banks and Rabinovici [3] (see also [1]), the lowest fermion bound states obtained from $\psi(2,1)$ and $\psi(2,3)$ by $s u(2)$ confinement are expected from their binding with those scalar framon fields carrying $s u(2)$ indices, namely the "weak" framons $\phi_{r}^{\tilde{r} \tilde{a}}$, thus:

$$
\begin{aligned}
\chi^{\tilde{r} \tilde{a}} & =\sum_{r}\left(\phi_{r}^{\tilde{r} \tilde{a}}\right)^{*} \psi_{r} ; \\
\chi_{a}^{\tilde{r} \tilde{a}} & =\sum_{r}\left(\phi_{r}^{\tilde{r} \tilde{a}}\right)^{*} \psi_{r a} .
\end{aligned}
$$

These are identified in the electroweak theory as respectively left-handed leptons and quarks, which assignment we here also adopt.

If we take the confinement picture as actually physical, and not merely as an alternative presentation of the conventional symmetry-breaking picture in the electroweak theory, then, just as in the case of boson bound states formed from framons considered before, there can in principle be many other fermion states obtainable as excitations of leptons and quarks from binding the fundamental fermions $\psi(2,1), \psi(2,3)$ with framons via $s u(2)$ confinement. At the moment, however, for our immediate purpose of deducing Standard Model properties from the constructed framework, we shall be concerned only with the leptons and quarks given explicitly above as the lowest fermion bound states.

The first thing of interest to note in the above expressions is that the bound states, in contrast to the fundamental fermions $\psi$ 's, now carry global indices, $\tilde{r}, \tilde{a}$ and indeed also $\tilde{q}$ not yet exhibited, which they acquire through their framon constituents. In particular, the $\tilde{r}$ indices for the global $\widetilde{s u}(2)$ symmetry, which occurred already in the pure electroweak theory treated by, for example 't Hooft [6], was taken there as representing up-down flavour. Explicitly, for the present scheme, if we take $\tilde{r}=\mp$, we would have, with $\psi_{r}=\psi_{r}^{(-1 / 2)}$ in (6.4), just by adding up the charges from their constituents, the following $u(1)$ and $\tilde{u}(1)$ charges $[q, \tilde{q}]$ for the bound states $\chi$,

$$
\left(\begin{array}{l}
\chi^{(-) \tilde{a}} \\
\chi^{(+) \tilde{a}}
\end{array}\right) \sim\left(\begin{array}{c}
{\left[0,-\frac{1}{2}\right]} \\
{\left[-1, \frac{1}{2}\right]}
\end{array}\right) ; \quad\left(\begin{array}{c}
\chi_{a}^{(-) \tilde{a}} \\
\chi_{a}^{(+) \tilde{a}}
\end{array}\right) \sim\left(\begin{array}{c}
{\left[\frac{2}{3},-\frac{1}{2}\right]} \\
{\left[-\frac{1}{3}, \frac{1}{2}\right]}
\end{array}\right) .
$$


We see that we obtain in the first doublet (of $\widetilde{s u}(2)$ ) exactly the right $u(1)$ charges for the leptons $\left(\nu, e^{-}\right)$, and for the second doublet those for the quarks $(u, d)$.

What is new, however, is that the leptons and quarks have now acquired, in addition to flavour, a charge $\tilde{q}$ for the global $\tilde{u}(1)$ as well as an index $\tilde{a}$ referring to the global $\widetilde{s u}(3)$ symmetry. As already indicated in the introduction, assumption (C), we would associate the $\widetilde{s u}(3)$ symmetry with fermion generations, a theme which we shall develop further in the rest of the present paper. For the moment, let us first ask what is the physical meaning, if any, of the $\tilde{u}(1)$ symmetry and charge.

The theory having been constructed to be invariant under both $u(1)$ and $\tilde{u}(1)$ by virtue of the framon hypothesis, it follows that both the $u(1)$ charge $q$ and the $\tilde{u}(1)$ charge $\tilde{q}$ are conserved quantities. This ought then to imply another conserved quantity in addition to the electric charge $q$. However, when applied to framons where the $\tilde{u}(1)$ charge originates, it gives nothing new, since $\tilde{q}$ there is always equal in magnitude and opposite in sign to the electric charge $q$ so that the conservation of one necessarily implies the conservation of the other. But such a situation will not in general be maintained when applied to compound particles formed from framons with other fields, which carry only the charge $q$, not the charge $\tilde{q}$. An examination of (5.4), however, shows that for the boson states $H, W^{ \pm}$, and $\gamma-Z$, the condition $\tilde{q}=-q$ still obtains so that again no new conserved quantity appears. This is because these boson states are formed from a framon-antiframon pair, some with a gauge boson $B_{\mu}$ which carries neither charge. But with leptons and quarks formed from a fundamental charged fermion with a single framon field, the condition $\tilde{q}=-q$ no longer applies. Instead, as seen in (6.6), the value of $\tilde{q}$ is shifted from $-q$ as follows:

$$
\tilde{q}=-q+\frac{1}{2}(B-L)
$$

where the special amount of the shift can be traced eventually to the charge assignments to the fundamental fermion fields, which in turn arise from the choice of $U(1,2,3)$ as gauge group. That being the case, the conservation of both $q$ and $\tilde{q}$ as required by the theory now implies not just the conservation of the electric charge $q$ but also the conservation of the baryon-lepton number $B-L$.

It thus seems that the new global $\tilde{u}(1)$ invariance obtained through implementing the framon idea has indeed a physical meaning, and a rather important one, namely baryon-lepton number conservation. Now baryon number 
conservation, or in its modern form as $B-L$ conservation, is a mystery which has puzzled us particle physicists for some time. As long ago as the 1950's, Lee and Yang [14] already asked the searching question why we knew of no gauge invariance principle which implies baryon number conservation, although it is one of the best kept conservation law known in nature. It is thus very interesting that the framon idea under consideration provides now an answer for it. In the framon scheme, baryon number (or $B-L$ in modern terms) is "dual" to the electric charge in a similar sense as flavour $\widetilde{s u}(2)$ is "dual" to the confining local gauge symmetry $s u(2)$, or, as generations is "dual" to su(3) colour, as we hope next to show.

As already noted, the compound fields $\chi$ in (6.5) carry also an $\tilde{a}$ index acquired from their framon constituents which means in physical terms that our left-handed leptons and quarks should each exist in $\widetilde{s u}(3)$ (anti-)triplets. Since the theory is invariant under $\widetilde{s u}(3)$ one would need in any case to account for the existence of this multiplet structure as one did for the $\tilde{u}(1)$ charge. It seems thus natural to try associating it with fermion generations, for which there has been wanting a plausible explanation already for decades.

To see whether such a supposition might work, let us try first to construct Yukawa couplings for the fermions in (6.1) with the "weak" framon fields. In analogy to the standard electroweak theory, we suggest the following:

$$
\begin{aligned}
\mathcal{A}_{\mathrm{YK}}= & \sum_{[\tilde{a}][b]} Y_{[b]}^{\text {lepton }} \bar{\psi}_{[\tilde{a}]}^{r} \phi_{r}^{(-) \tilde{a}} \frac{1}{2}\left(1+\gamma_{5}\right) \psi^{[b]}+\sum_{[\tilde{a}][b]} Y_{[b]}^{\text {lepton }} \bar{\psi}_{[\tilde{a}]}^{r} \phi_{r}^{(+) \tilde{a}} \frac{1}{2}\left(1+\gamma_{5}\right) \psi^{\prime[b]} \\
& + \text { h.c. }
\end{aligned}
$$

for leptons, and

$$
\begin{aligned}
\mathcal{A}_{\mathrm{YK}}= & \sum_{[\tilde{a}][b]} Y_{[b]}^{\text {quark }} \bar{\psi}_{[\tilde{a}]}^{r a} \phi_{r}^{(-) \tilde{a}} \frac{1}{2}\left(1+\gamma_{5}\right) \psi_{a}^{[b]}+\sum_{[\tilde{a}][b]} Y_{[b]}^{\text {quark }} \bar{\psi}_{[\tilde{a}]}^{r a} \phi_{r}^{(+) \tilde{a}} \frac{1}{2}\left(1+\gamma_{5}\right) \psi_{a}^{\prime}[b] \\
& + \text { h.c. }
\end{aligned}
$$

for quarks, where the indices in brackets $[\tilde{a}]$ and $[b]$ label just 3 identical copies of the same fields. These expressions are by construction invariant under Lorentz transformations and the internal symmetries $s u(2)$ and $s u(3)$. Invariance under $\widetilde{s u}(2)$ can be seen by recalling from (2.15) the expressions of $\phi_{r}^{( \pm)}$as the inner products $\boldsymbol{\alpha}^{( \pm)} \cdot \boldsymbol{\phi}_{r}$ between $2 \widetilde{s u}(2)$ vectors. What remains to be checked is then just the invariance with respect to $u(1), \tilde{u}(1)$ and $\widetilde{s u}(3)$. Invariance under $u(1)$ can be guaranteed by assigning appropriate $u(1)$ charges to the right-handed fields, thus $q=0$ for $\psi^{[b]}, q=-1$ for $\psi^{\prime[b]}$, 
$q=2 / 3$ for $\psi_{a}^{[b]}$, and $q=-1 / 3$ for $\psi_{a}^{\prime}[b]$, namely the same charges as the corresponding compound state $\chi$ in each case. This procedure is the same as in the conventional formulation of the Standard Model. We only need to check here that these charge assignments to the right-handed fields are consistent with those allowed by the gauge group $U(1,2,3)$ as listed in (2.9), and one sees that they are. Similarly, $\tilde{u}(1)$ invariance can be ensured also by assigning to the right-handed fields the same $\tilde{u}(1)$ charges $\tilde{q}$ as their corresponding compound states. This contravenes in principle our rule that fundamental fermions should not carry global charges or indices. This is however just a matter of convenience, for these charges being global can equally be absorbed into the Yukawa couplings $Y_{[b]}$ etc. We follow the standard convention and assign the $\tilde{u}(1)$ charge to the right-handed field so that they carry the same baryon and lepton number as their left-handed partners and so guarantee $\tilde{u}(1)$ invariance. There remains then only $\widetilde{s u}(3)$ invariance, of which, strictly speaking, the expressions (6.8) and (6.9) do not have. Under an $\widetilde{s u}(3)$ transformation, the framons $\phi$ transform but the left-handed fermions $\psi^{[\tilde{a}]}$ with which the $\tilde{a}$ index of the framons are contracted, do not transform. However, if we force a transformation on these latter fields, it would mean only a relabelling of these identical fields, and physics should not be affected. Thus $\widetilde{s u}(3)$ invariance is maintained in this more specialized sense. This is similar to the argument used below, by relabelling the right-handed fields, to cast the fermion mass matrix into a hermitian form often found useful in the usual Standard Model. We thus propose to accept the above Yukawa couplings as valid on this basis.

Suppose we do, then on substituting the vacuum expectation value $\zeta_{W}$ of the weak framon field into the Yukawa couplings above, one obtains mass matrices of the form:

$$
m \sim\left(\begin{array}{c}
\alpha^{\tilde{1}} \\
\alpha^{\tilde{2}} \\
\alpha^{\tilde{3}}
\end{array}\right)\left(Y_{[1]}, Y_{[2]}, Y_{[3]}\right) \frac{1}{2}\left(1+\gamma_{5}\right)+\left(\begin{array}{c}
Y_{[1]}^{*} \\
Y_{[2]}^{*} \\
Y_{[3]}^{*}
\end{array}\right)\left(\alpha^{\tilde{1} *}, \alpha^{\tilde{2} *}, \alpha^{\tilde{3} *}\right) \frac{1}{2}\left(1-\gamma_{5}\right) .
$$

Equivalently, by relabelling the right-handed fields appropriately, we can recast the mass matrix in a hermitian form with no $\gamma_{5}$ [15] as:

$$
m=m_{T}\left(\begin{array}{c}
\alpha^{\tilde{1}} \\
\alpha^{\tilde{2}} \\
\alpha^{\tilde{3}}
\end{array}\right)\left(\alpha^{\tilde{1} *}, \alpha^{\tilde{2} *}, \alpha^{\tilde{3} *}\right) .
$$

In other words, we obtain that the mass matrix of all quarks and leptons are 
similar, being just a product of the same vector $\boldsymbol{\alpha}$ in generation space by its hermitian conjugate, and differ for different fermion species, i.e. whether lepton or quark or whether flavour up or down, only by the normalization:

$$
m_{T}=\zeta_{W} \rho_{T}^{2}
$$

with

$$
\rho_{T}^{2}=\left|Y_{[1]}\right|^{2}+\left|Y_{[2]}\right|^{2}+\left|Y_{[3]}\right|^{2} .
$$

This form for the fermion mass matrix plays a very significant role in our understanding of the fermion mass hierarchy and mixing pattern observed in experiment, a theme that will be taken up again in the next section.

Before we do so, let us first make a necessary detour to consider the soft hadron scenario where colour $s u(3)$ as well confines. As remarked before, we are not at present interested in details of the hadronic spectrum and will not therefore examine in general terms the many fermion states which are obtained by confining via colour the fundamental fermion fields introduced above with the scalar framon fields. We shall only, for a reason which will be made clear later, work out a special case as example. For our purpose, it will be sufficient to consider the (hadronic) fermion states obtained by confining $\psi_{a}$ via colour confinement with the "strong" framon from which we shall even omit the $\tilde{r}$ index as being here inessential and write it as $\phi_{a}^{\tilde{a}}$.

Our first task is to construct a Yukawa coupling term for $\psi_{a}$ with $\phi_{a}^{\tilde{a}}$. We notice that whereas the framon field carries an $\tilde{a}$ index for $\widetilde{s u}(3)$, the fermion fields do not. To maintain $\widetilde{s u}(3)$ invariance, therefore, we need here a vector in $\widetilde{s u}(3)$ space to saturate this $\tilde{a}$ index. As noted already in [1, there is no such vector available to play this role within the purely strong sector. But in the present set-up, there is the vector $\boldsymbol{\alpha}$ coming from the weak sector which can possibly be so employed. In thus introducing a vector originating from the weak sector so as to construct a Yukawa term in the strong sector, one is imitating, in spirit though not in detail, the previous construction of the Yukawa terms in the weak sector (6.8) and (6.9) by means of the $\widetilde{s u}(2)$ vectors $\boldsymbol{\alpha}^{( \pm)}$originating from the electromagnetic $u(1)$ sector so as to guarantee $\widetilde{s u}(2)$ invariance, and in that case, one obtained the standard electroweak theory result. The vector $\boldsymbol{\alpha}$, however, does not have a definite value, but, the vacuum being degenerate as asserted previously, can point in any direction in $\widetilde{s u}(3)$ space. Nevertheless, these directions being all gauge equivalent, it should not matter which particular value we choose. Let us then just select an arbitrary direction, say, $\boldsymbol{\alpha}_{0}$ and construct our Yukawa 
term as follows:

$$
\mathcal{A}_{\mathrm{YK}}=\sum_{[b]} Z_{[b]} \bar{\psi}^{a} \boldsymbol{\phi}_{a} \cdot \boldsymbol{\alpha}_{0} \frac{1}{2}\left(1+\gamma_{5}\right) \psi^{[b]}+\text { h.c. }
$$

where $\psi^{[b]}, b=1,2,3$ are again just 3 copies of right-handed fields. This has then all the invariance properties that are required and seems to be the only Yukawa term with these properties that one can construct in the strong sector from the quantities so far introduced. What the chosen value for $\boldsymbol{\alpha}_{0}$ represents will become clear later as the formalism develops.

Again, for consequences of such a Yukawa term, because of the distortion of the strong framon vacuum from orthonormality, the working out is a little complicated, and will be given here in detail only for the 2-G model. In that case, for any $\boldsymbol{\alpha}_{0}$ that we may have chosen, the theory being invariant under $\widetilde{s u}(2)$, we can always choose to work in the gauge where $\boldsymbol{\alpha}_{0}$ points in the up direction and is real, thus:

$$
\boldsymbol{\alpha}_{0}=\left(\begin{array}{l}
1 \\
0
\end{array}\right)
$$

Further, the theory being also invariant locally under $s u(2)$, we can also choose to work in the local $s u(2)$ gauge where the vacuum value for the framon field $\Phi$ corresponding to this $\boldsymbol{\alpha}_{0}$ is triangular, which in this case actually means diagonal, as given in (4.40) above. We shall refer henceforth to this chosen gauge as the $T_{0}$-gauge.

Our next task is to evaluate the mass matrix for the fermion states formed from $\psi_{a}$ and $\phi_{a}^{\tilde{a}}$ by colour confinement. As for the leptons and quarks in the weak sector, the mass matrix for these is obtained by substituting for $\Phi$ in the Yukawa term its vacuum value. For the general vacuum labelled by $\boldsymbol{\alpha}$, the vacuum value for $\Phi$ is given already in (4.6), with $\delta$ and $\Delta$ given in terms of $\alpha$ in (4.18). This is, however, in the $s u(2)$ gauge, say the $T$-gauge, where the vacuum value of $\Phi$ is triangular, and this gauge changes from vacuum to vacuum labelled by different values of $\boldsymbol{\alpha}$. In what follows we shall be interested in comparing the mass matrix, and hence the vacuum values of $\Phi$ at different $\boldsymbol{\alpha}$ 's and this will make sense only if we keep the same gauge, let us say the $T_{0}$-gauge defined above. The value of $\Phi$ at the vacuum labelled by $\boldsymbol{\alpha}$ in the $T_{0}$ gauge can be obtained either by operating from the left on (4.6) with $\Theta^{-1}$ for $\Theta$ in (4.44), changing thus from the original $T$-gauge to the $T_{0}$-gauge we prefer, or else by operating from the right on (4.40) with $A^{-1}$ for $A$ in (4.41), i.e. translating the vacuum value at the reference point $\boldsymbol{\alpha}_{0}$ to the general point $\boldsymbol{\alpha}$. In either case, one obtains for the vacuum value 
of $\Phi$ at $\boldsymbol{\alpha}$ as (4.43), which we rewrite here again for future convenience:

$$
V_{0}(\boldsymbol{\alpha})=\Phi_{V A C}\left(\boldsymbol{\alpha}_{0}\right) A^{-1}=\left(\begin{array}{cc}
\sqrt{\frac{1+R}{2}} \cos \alpha e^{i \beta} & \sqrt{\frac{1+R}{2}} \sin \alpha e^{i \gamma} \\
-\sqrt{\frac{1-R}{2}} \sin \alpha e^{-i \gamma} & \sqrt{\frac{1-R}{2}} \cos \alpha e^{-i \beta}
\end{array}\right)
$$

Substituting the vacuum value (6.16) of $\Phi$ into (6.14), one obtains then for the fermion bound states via $s u(2)$ (2-G colour) confinement formed from $\psi_{a}$ with $\phi_{a}^{\tilde{a}}$ the mass matrix:

$$
\mathbf{m}=\zeta_{s}\left(V_{0} \boldsymbol{\alpha}_{0}\right)\left(Z_{[1]}, Z_{[2]}\right) \frac{1}{2}\left(1+\gamma_{5}\right)+\left(\begin{array}{c}
Z_{[1]}^{*} \\
Z_{[2]}^{*}
\end{array}\right)\left(V_{0} \boldsymbol{\alpha}_{0}\right)^{\dagger} \frac{1}{2}\left(1-\gamma_{5}\right),
$$

where we have used a bold-faced letter $\mathbf{m}$ to remind ourselves that we are dealing here with hadron states and to distinguish it from the mass matrix $m$ introduced above for leptons and quarks. Also, to save on the notation, we have omitted from $V_{0}$ its argument, leaving its dependence on $\boldsymbol{\alpha}$ understood, as we shall do as well for related quantities in future. Again, as was done before for (6.10), by appropriately relabelling the right-handed singlet fermions $\psi^{[b]}$, we can rewrite $\mathbf{m}$ in the hermitian form independent of $\gamma_{5}$ as:

$$
\mathbf{m}=\mathbf{m}_{T}\left|v_{0}\right\rangle\left\langle v_{0}\right|
$$

with

$$
\left|v_{0}\right\rangle=V_{0} \boldsymbol{\alpha}_{0}
$$

Explicitly,

$$
\mathbf{m}_{T}=\zeta_{s} \rho_{S} ; \quad \rho_{S}=\sqrt{\left|Z_{[1]}\right|^{2}+\left|Z_{[2]}\right|^{2}}
$$

and:

$$
\left|v_{0}\right\rangle=\left(\begin{array}{c}
\sqrt{\frac{1+R}{2}} \cos \alpha e^{i \beta} \\
-\sqrt{\frac{1-R}{2}} \sin \alpha e^{-i \gamma}
\end{array}\right)
$$

For our future use, we need also to derive the couplings of these bounds state fermions to the Higgs bosons listed in the preceding section. These couplings are obtained by expanding the Yukawa coupling term (6.14) to first order in fluctuations of the framon fields about their vacuum values. With right-handed fermions labelled as in (6.18), these couplings for the fluctuations $\delta \phi_{a}^{\tilde{a}}$ are easily seen to be given just as:

$$
\begin{aligned}
\Gamma_{a R}^{\tilde{a}} & =\rho_{S}|v\rangle\left\langle v_{0}\left|\frac{1}{2}\left(1+\gamma_{5}\right)+\rho_{S}\right| v_{0}\right\rangle\langle v| \frac{1}{2}\left(1-\gamma_{5}\right) \\
\Gamma_{a I}^{\tilde{a}} & =i \rho_{S}|v\rangle\left\langle v_{0}\left|\frac{1}{2}\left(1+\gamma_{5}\right)-i \rho_{S}\right| v_{0}\right\rangle\langle v| \frac{1}{2}\left(1-\gamma_{5}\right),
\end{aligned}
$$


with:

$$
|v\rangle=V \boldsymbol{\alpha}_{0} ; \quad(V)_{b}^{\tilde{b}}=\delta_{a b} \delta^{\tilde{a} \tilde{b}} .
$$

What we need, however, are the couplings for the Higgs states $H_{1}, H_{2}$, $H_{R}, H_{I}$ identified in the preceding section. These were given as linear combinations of $\delta \phi_{a}^{\tilde{a}}$ in (5.9) in terms of the matrices $\widehat{V}_{K}$. But these were in the $T$-gauge which we have first to transform back into the selected $T_{0}$-gauge, thus:

$$
V_{K}=\Theta^{-1} \widehat{V}_{K}
$$

The corresponding coupling of the $K$ th Higgs state to the fermion states is then given as::

$$
\Gamma_{K}=\rho_{S}\left|v_{K}\right\rangle\left\langle v_{0}\left|\frac{1}{2}\left(1+\gamma_{5}\right)+\rho_{S}\right| v_{0}\right\rangle\left\langle v_{K}\right| \frac{1}{2}\left(1-\gamma_{5}\right)
$$

for $K=1,2, R, I$, where for $V_{K}$ listed as in (6.24):

$$
\begin{aligned}
\left|v_{1}\right\rangle & =V_{1} \boldsymbol{\alpha}_{0}=\left(\begin{array}{c}
\cos \theta e^{i \beta} \\
-\sin \theta e^{-i \gamma}
\end{array}\right) \\
\left|v_{2}\right\rangle & =V_{2} \boldsymbol{\alpha}_{0}=\frac{1}{\sqrt{x^{2} \cos ^{2} \delta+y^{2}}}\left(\begin{array}{c}
-x^{2} \sin \theta \sin \delta \cos \delta e^{i \beta} \\
-x^{2} \cos \theta \sin \delta \cos \delta e^{-i \gamma}
\end{array}\right), \\
\left|v_{R}\right\rangle & =V_{R} \boldsymbol{\alpha}_{0}=\frac{1}{\sqrt{x^{2} \cos ^{2} \delta+y^{2}}}\left(\begin{array}{c}
y \sin \theta e^{i \beta} \\
y \cos \theta e^{-i \gamma}
\end{array}\right) \\
\left|v_{I}\right\rangle & =V_{I} \boldsymbol{\alpha}_{0}=i\left(\begin{array}{c}
(-x \cos \theta \sin \delta-y \sin \theta) e^{i \beta} \\
(x \sin \theta \sin \delta-y \cos \theta) e^{-i \gamma}
\end{array}\right) .
\end{aligned}
$$

There is no difficulty of principle extending the above considerations to the actual 3-G situation. For the present, however, since the solution for the vacuum has only been found to leading order in the supposedly small parameter $R$, and the gauge relationship between them only worked out for a special class of $\widetilde{s u}(3)$ transformations $A$, the same restrictions remain in what follows. Apart from these restrictions, the formulae (6.18) - (6.19) above for the mass matrix of the analogous bound state remain essentially the same, only with the matrix $V_{0}$ being now the $3-\mathrm{G}$ version given in (4.52). The couplings of these fermion bound states to the Higgs fields also remain basically the same as in (6.25) although notation-wise we find it convenient to distinguish the 3 types of Higgs $K=k, k R, k I, k=1,2,3$ thus:

$$
\Gamma_{k}=\rho_{S}\left|v_{k}\right\rangle\left\langle v_{0}\left|\frac{1}{2}\left(1+\gamma_{5}\right)+\rho_{S}\right| v_{0}\right\rangle\left\langle v_{k}\right| \frac{1}{2}\left(1-\gamma_{5}\right)
$$




$$
\begin{aligned}
\Gamma_{k}^{R} & =\rho_{S}\left|w_{k}^{R}\right\rangle\left\langle v_{0}\left|\frac{1}{2}\left(1+\gamma_{5}\right)+\rho_{S}\right| v_{0}\right\rangle\left\langle w_{k}^{R}\right| \frac{1}{2}\left(1-\gamma_{5}\right), \\
\Gamma_{k}^{I} & =\rho_{S}\left|w_{k}^{I}\right\rangle\left\langle v_{0}\left|\frac{1}{2}\left(1+\gamma_{5}\right)+\rho_{S}\right| v_{0}\right\rangle\left\langle w_{k}^{I}\right| \frac{1}{2}\left(1-\gamma_{5}\right),
\end{aligned}
$$

where:

$$
\left|v_{k}\right\rangle=\Theta^{-1} \widehat{V}_{k} \boldsymbol{\alpha}_{0} ; \quad\left|w_{k}^{R}\right\rangle=\Theta^{-1} \widehat{W}_{k}^{R} \boldsymbol{\alpha}_{0} ; \quad\left|w_{k}^{I}\right\rangle=\Theta^{-1} \widehat{W}_{k}^{I} \boldsymbol{\alpha}_{0}
$$

for $\widehat{V}_{k}, \widehat{W}_{k}^{R}, \widehat{W}_{k}^{I}$ listed in (5.14). The resulting formulae for these vectors are a little complicated even to first order in $R$. Since the algebra is straightforward, we shall give the answer here only for $\left|v_{0}\right\rangle$ :

$$
\left|v_{o}\right\rangle=\left(\begin{array}{l}
\sqrt{\frac{1+2 R}{3}} c_{2} c_{3} e^{i \beta_{1}} \\
-\sqrt{\frac{1-R}{3}} s_{3} e^{i\left(\beta_{1}+\sigma_{3}\right)} \\
-\sqrt{\frac{1-R}{3}} s_{2} c_{3} e^{-i\left(\beta_{2}+\beta_{3}+\sigma_{3}\right)}
\end{array}\right),
$$

which is just the first column of the matrix $V_{0}$ in (4.52) and will be of use later.

\section{The Rotating Fermion Mass Matrix}

By a rotating mass matrix here we mean one which changes its orientation in generation space with changing scale, in much the same way as a running coupling changes with changing scale its value. A coupling changes with scale because of renormalization effects; so in the same way a mass matrix may change its orientation in generation space. It is in fact quite easy to imagine or construct situations in which it does do so.

The reason we are interested in a rotating fermion mass matrix is that it has been suggested that this may lead to mass hierarchy and mixing patterns for fermions similar to what have been observed in experiment. Indeed, one can even claim there is circumstantial evidence that the observed mass and mixing patterns for leptons and quarks are due to a rotating fermion mass matrix [16]. Furthermore, a phenomenological model (DSM) has been constructed which gives a good description of these phenomena, including in particular neutrino oscillations, depending on only a small number of parameters [7, 4].

Starting anew from more considered premises, we have now constructed, to us, a much more theoretically attractive and internally consistent model. 
We naturally wish to ask whether in this new model the fermion mass matrix will still rotate and give rise to a similar explanation for the mass and mixing patterns. At first sight, this might seem difficult, since the fermions we are interested in are the leptons and quarks which have rather little structure in our new model, being bound states via the deeply confining $s u(2)$ symmetry, and should appear at present experimental conditions as approximate point particles. Although they are made to carry a generation index in view of the weak framon field which is one of their constituents and which carries this index, this index is only global and brings with it no new interactions. Indeed, this is in a sense fortunate for one would not want, and probably cannot admit, any blatant new interactions for leptons and quarks for these may spoil the already good description of these particles by the Standard Model. For this reason, in the lepton and quark mass matrix given above in (6.11), the generation index appeared only in the global factor $\boldsymbol{\alpha}$ which has no explicit coupling to any gauge field.

However, the interesting thing is that this vector $\boldsymbol{\alpha}$ is coupled nevertheless to the strong sector via the framon potential constructed to satisfy the symmetries intrinsic to the system. Indeed, as shown above in section 4 , the vacuum of the framon potential is degenerate and depends on the direction of $\boldsymbol{\alpha}$ so that if the vacuum moves from one value to another within the degenerate set, which it can do under renormalization effects in the strong sector, then $\boldsymbol{\alpha}$ can change in direction, i.e. in other words, rotate. The attention is then shifted to the behaviour of the vacuum under renormalization, which we shall now study.

The vacuum value of the framon field $\Phi$, we noticed above, occurs in the mass matrix $\mathbf{m}$ for the hadron bound state in (6.18) via (6.19). We can thus obtain information on the change in value of the vacuum value of $\Phi$, and hence on the rotation of $\boldsymbol{\alpha}$, by studying the behaviour of $\mathbf{m}$ in (6.18) under renormalization. Now a similar problem has been studied before in the phenomenological model DSM [8], where it was found that the rotation, if any, would come mostly from insertions of Higgs loops in the fermion propagator (i.e. for the hadronic bound state fermions, in the present language), the effect of other loops being suppressed. It is for this reason that we have worked out above in some detail the spectra of these fermion and Higgs bound states as well as their couplings. With these results, we can now calculate the effect of Higgs loop insertions, which we shall do below to 1-loop order. 
The insertion of a Higgs loop to the fermion self-energy is of the form:

$$
\Sigma(p)=\frac{i}{(4 \pi)^{4}} \sum_{K} \int d^{4} k \frac{1}{k^{2}-M_{K}^{2}} \Gamma_{K} \frac{(\not p-\not h)+\mathbf{m}}{(p-k)^{2}-\mathbf{m}^{2}} \Gamma_{K},
$$

where we may for the moment take $K$ to label the Higgs mass eigenstates. After standard manipulations, regularizing the divergence by dimensional regularization, one obtains:

$$
\Sigma(p)=-\frac{1}{16 \pi^{2}} \sum_{K} \int_{0}^{1} d x \Gamma_{K}\left\{\bar{C}-\ln \left(Q^{2} / \mu^{2}\right)\right\}[\not p(1-x)+\mathbf{m}] \Gamma_{K},
$$

where

$$
Q^{2}=\mathbf{m}^{2} x+M_{K}^{2}(1-x)-p^{2} x(1-x),
$$

with $\bar{C}$ being the divergent constant to be subtracted in the standard $\overline{\mathrm{MS}}$ scheme. The renormalization to the mass matrix $\delta \mathbf{m}$ is obtained by first commuting the $\not p$ in the numerator half to the extreme left and half to the extreme right, then putting $\not p=\mathbf{m}$ and $p^{2}=\mathbf{m}^{2}$. The full explicit expression for $\delta \mathbf{m}$ so obtained together with more details of the calculation can be found in [17]. Here, we are interested only in the terms dependent on the scale $\mu$. These are of two types. First, there are terms of the form:

$$
\Gamma_{K} \mathbf{m} \Gamma_{K}=\rho_{S}^{2}\left\langle v_{0} \mid v_{0}\right\rangle\left\langle v_{0} \mid v_{K}\right\rangle\left|v_{K}\right\rangle\left\langle v_{0}\right| \frac{1}{2}\left(1+\gamma_{5}\right)+\text { c.c. }
$$

Then there are terms of the form:

$$
\begin{aligned}
& \Gamma_{K} \not p \Gamma_{K} \rightarrow \\
& \quad \frac{1}{2} \rho_{S}^{2}\left\{\left\langle v_{0} \mid v_{0}\right\rangle\left\langle v_{K} \mid v_{0}\right\rangle\left|v_{K}\right\rangle\left\langle v_{0}\left|+\left\langle v_{0} \mid v_{0}\right\rangle\left\langle v_{K} \mid v_{K}\right\rangle\right| v_{0}\right\rangle\left\langle v_{0}\right|\right\} \frac{1}{2}\left(1+\gamma_{5}\right) \\
& \quad+\text { c.c. }
\end{aligned}
$$

In (7.4) and (7.5), we have already commuted $\not p$ to the left and right as stipulated and used the known forms for the tree-level mass matrix (6.18) and Higgs couplings (6.25).

We notice that the second term in (7.5) is proportional to the tree level mass matrix (6.18), and will thus only change its normalization, not its orientation. Any rotation will thus have to come from the other terms, either of which will add a correction of the form:

$$
\sum_{K}\left\langle v_{0} \mid v_{K}\right\rangle\left|v_{K}\right\rangle
$$


or

$$
\sum_{K}\left\langle v_{K} \mid v_{0}\right\rangle\left|v_{K}\right\rangle
$$

to the vector $\left|v_{0}\right\rangle$ on the left, and will change the orientation of the mass matrix, provided of course that these corrections are neither vanishing nor parallel to $\left|v_{0}\right\rangle$ itself. For this reason, we call (7.6) and (7.7) the governing vectors for rotation, and it is on these now that our attention is turned.

Again, to avoid getting bogged down by algebraic complications too soon, we shall work out first the governing vectors in the $2-\mathrm{G}$ model. We recall that the index $K$ is meant here to label the Higgs mass eigenstates, not the same as in (6.26). This presents no difficulty in principle, for we had the Higgs mass matrix in (5.11), which if necessary can be diagonalized to give the mass eigenstates as orthogonal transforms of the vectors in (5.10), from which eigenstates, the corresponding $\left|v_{K}\right\rangle$ can be recalculated. However, this will not be necessary since an orthogonal transformation among the vectors $V_{K}$ will leave the governing vectors invariant. We can thus evaluate the governing vectors with just the old set of $\left|v_{K}\right\rangle$ in (6.26). The answer is as follows. First, we notice by (4.46) or (4.47) that both $\left|v_{2}\right\rangle$ and $\left|v_{R}\right\rangle$ are orthogonal to $\left|v_{0}\right\rangle$ giving thus no contributions, while $\left|v_{1}\right\rangle$ is parallel to $\left|v_{0}\right\rangle$. Hence, summing over the "real" $K$ 's, we have:

$$
\sum_{K=1,2, R}\left\langle v_{0} \mid v_{K}\right\rangle\left|v_{K}\right\rangle=\sum_{K=1,2, R}\left\langle v_{K} \mid v_{0}\right\rangle\left|v_{K}\right\rangle=\left|v_{0}\right\rangle .
$$

This means that the "real" states will give no rotation to the mass matrix. The "imaginary" state $K=I$, on the other hand, gives the following contribution:

$$
|\operatorname{gvI}\rangle=\left\langle v_{0} \mid v_{I}\right\rangle\left|v_{I}\right\rangle=-\left\langle v_{I} \mid v_{0}\right\rangle\left|v_{I}\right\rangle=-\frac{1}{2} R \sin 2 \alpha\left(\begin{array}{c}
\sqrt{\frac{1+R}{2}} \sin \alpha e^{i \beta} \\
\sqrt{\frac{1-R}{2}} \cos \alpha e^{-i \gamma}
\end{array}\right),
$$

where we have used the relations (4.46) and (4.18) to simplify the expression to the given form. This being in general neither vanishing nor parallel to $\left|v_{0}\right\rangle$, we conclude that in the $2-\mathrm{G}$ case, the vector $\left|v_{0}\right\rangle$ will indeed in general rotate with changing scale as we hope, and that by section 4 above it will drag the vector $\boldsymbol{\alpha}$ along with it.

Indeed, substituting the result (7.9) into (7.2), one obtains the following renormalization group equation for the rotating vector $\left|v_{0}\right\rangle$ :

$$
\frac{d}{d\left(\ln \mu^{2}\right)}\left|v_{0}\right\rangle=-\frac{3}{64 \pi^{2}} \rho_{S}^{2}|\operatorname{gvI}\rangle,
$$


where one has neglected terms which changes only the normalization of the vector which is not of immediate interest here. The equation makes the rotation effect with respect to scale change explicit. In particular, one notes that at $\alpha=n \pi / 2$, the governing vector vanishes. This means that these are fixed points of the rotation which will be of special significance later when practical applications to fermion mixing are considered.

To visualize more easily the behaviour of the rotating vectors between fixed points, let us simplify the equation by taking the leading order in an expansion in the supposedly small parameter $R$, in which case we have:

$$
|\mathrm{gvI}\rangle \sim-\frac{1}{2} R \sin 2 \alpha\left(\begin{array}{c}
\sin \alpha e^{i \beta} \\
\cos \alpha e^{-i \gamma}
\end{array}\right),
$$

of first order in smallness and orthogonal to $\left|v_{0}\right\rangle$. Suppose we start with $\boldsymbol{\alpha}$ in the first quadrant, i.e. $0<\alpha<\pi / 2$, which gives then, according to (6.19) a $\left|v_{0}\right\rangle$ in the 4th quadrant. Then, according to the rotation equation (7.10) and the expression (7.11) for $|\mathrm{gvI}\rangle$, a change in the scale $\mu$ by a positive amount will give a vector increment to $\left|v_{0}\right\rangle$ pointing back in the first quadrant. This means that for increasing $\mu,\left|v_{0}\right\rangle$ will rotate in the counterclockwise direction, or that $\boldsymbol{\alpha}$ will rotate in the clockwise direction. Hence, on increasing $\mu$ further, $\alpha$ will eventually reach the limiting value 0 , or $\boldsymbol{\alpha}$ the limiting value $\boldsymbol{\alpha}_{0}=(1,0)$ which we have already noted to be a rotational fixed point. Recalling here that $\boldsymbol{\alpha}_{0}$ was the arbitrary value of $\boldsymbol{\alpha}$ that we had chosen to construct our Yukawa term (6.14), we now realize what it represents, namely the high scale limit of $\boldsymbol{\alpha}$, i.e. when $\mu \longrightarrow \infty$. Conversely, for decreasing $\mu$, $\boldsymbol{\alpha}$ will rotate in the counterclockwise direction reaching eventually at $\mu=0$ the fixed point at $\alpha=\pi / 2$, i.e. $\boldsymbol{\alpha}=(0,1)$.

These results are easily generalized. For example, had we started with an $\boldsymbol{\alpha}$ pointing in the 4 th quadrant, i.e. $-\pi / 2<\alpha<0$, then $\boldsymbol{\alpha}$ will rotate clockwise for increasing $\mu$, so that $\boldsymbol{\alpha}=(1,0)$ will remain the high scale fixed point, but $\boldsymbol{\alpha}$ will reach eventually instead at $\mu=0$ the fixed point at $\alpha=-\pi / 2$ or $\boldsymbol{\alpha}=(0,-1)$. Furthermore, given the intrinsic $\widetilde{s u}(2)$ invariance of the system, it is clear that the choice of the starting point $\boldsymbol{\alpha}_{0}$ has no particular meaning. One could have started with any other choice for $\boldsymbol{\alpha}_{0}$ to construct the Yukawa coupling, which $\boldsymbol{\alpha}_{0}$ will then play the role of the high scale fixed point, and $\boldsymbol{\alpha}$ will rotate for decreasing $\mu$, in either direction depending on the initial condition, for a quarter circle till it reaches the low scale fixed point at $\mu=0$.

The same analysis can be carried out for the actual 3-G case using the Higgs states listed in (5.14). This can and has been done at present to leading 
order in $R$ and for the special class of vacua defined by $A$ of (4.51). Again, as in the $2-\mathrm{G}$ case, one finds that all the "real" states $\left|v_{i}\right\rangle,\left|w_{i}^{R}\right\rangle$ are orthogonal, except for $\left|v_{1}\right\rangle$ which is parallel, to $\left|v_{0}\right\rangle$, so that their total contribution to the governing vector is just:

$$
\sum_{i}\left\langle v_{0} \mid v_{i}\right\rangle\left|v_{i}\right\rangle+\sum_{i}\left\langle v_{0} \mid w_{i}^{R}\right\rangle\left|w_{i}^{R}\right\rangle=\sum_{i}\left\langle v_{i} \mid v_{0}\right\rangle\left|v_{i}\right\rangle+\sum_{i}\left\langle w_{i}^{R} \mid v_{0}\right\rangle\left|w_{i}^{R}\right\rangle=\left|v_{0}\right\rangle,
$$

giving thus no rotation. On the other hand, one has from the "imaginary" states:

$$
\sum_{i}\left\langle v_{0} \mid w_{i}^{I}\right\rangle\left|w_{i}^{I}\right\rangle=-\sum_{i}\left\langle w_{i}^{I} \mid v_{0}\right\rangle\left|w_{i}^{I}\right\rangle=|\mathrm{gvI}\rangle
$$

with

$$
|\mathrm{gvI}\rangle=-\frac{\sqrt{3}}{4} R \sin 2 \theta\left(\begin{array}{c}
\sin \theta e^{i \beta_{1}} \\
\frac{\cos \theta \sin \phi}{\sqrt{1-\sin ^{2} \theta \cos ^{2} \phi}} e^{i\left(\beta_{1}+\sigma_{3}\right)} \\
\frac{\cos \phi \cos ^{2} \theta}{\sqrt{1-\sin ^{2} \theta \cos ^{2} \phi}} e^{-i\left(\beta_{2}+\beta_{3}+\sigma_{3}\right)}
\end{array}\right) .
$$

Again, this being neither parallel nor orthogonal to $\left|v_{0}\right\rangle$, there will in general be rotation, except when $\sin 2 \theta=0$ where the rotation vanishes and we have again rotational fixed points.

We shall leave the detailed analysis of the rotation for the 3-G case for later when the calculation has been made more complete. We note only that in restricting $A$ only to the form (4.51), we have thrown away a phase which could be important for the understanding of CP-violation in the mixing of quarks and leptons, which has so far not made an appearance. However, this is only a matter of algebraic complications which we need to sort out in the near future.

Given the rather significant role that the rotating fermion mass matrix will play in what follows, it would seem worthwhile to give here a brief resumé of the manner it arises in the present framework, which is surprisingly intricate, involving, as it does, the doubled invariance introduced by the framon idea in an essential way as well as a delicate interplay between the electroweak and strong sectors of the theory. The mass matrix here being of the factorized form (6.11), its rotation is encapsulated in the rotation of the vector $\boldsymbol{\alpha}$. 
This last is a vector in $\widetilde{s u}(3)$ space and originates in the weak framon $\phi_{r}^{\tilde{a} \tilde{r}}$ of (2.10), having at first nothing to do with the fundamental fermions (6.1). It only got attached to the left-handed bound fermion states $\chi^{\tilde{a} \tilde{r}}$ and $\chi_{a}^{\tilde{a} \tilde{r}}$ of (6.4) and (6.5) through their framon constituents, and so appear in the fermion mass matrix (6.11). This vector $\boldsymbol{\alpha}$, carrying only the global $\widetilde{s u}(3)$ index, has by itself no gauge interaction to give it rotation, but by virtue of its coupling to the strong sector via the linkage $\nu_{2}$ term in the framon potential (4.1), it feels the effects of strong dynamics. In passing, we note that this $\nu_{2}$ term in the framon potential was not introduced by fiat but is a consequence of the doubled invariance intrinsic to the framon idea. Apart from coupling $\boldsymbol{\alpha}$ to the strong sector, this same $\nu_{2}$ term also distorts the strong framon vacuum from its original simple configuration of an orthonormal triad to a configuration where deviations from both orthogonality and normality appear. The vacuum becomes degenerate under these distortions with deviations from orthogonality traded off with deviations from normality. And the vector $\boldsymbol{\alpha}$ is coupled to the strong sector in such a way that as the vacuum moves among the degenerate set, the vector $\boldsymbol{\alpha}$ moves (rotates) with it. That the vacuum does indeed move, or that the vector $\boldsymbol{\alpha}$ does indeed rotate, was finally ascertained by studying the renormalization effects to 1-loop order on the (hadron) fermion self-energy. Even in this, the interplay between the electroweak and strong sectors plays an essential role, for without the vector $\boldsymbol{\alpha}$ coming from the electroweak sector, one finds that one cannot even construct a Yukawa term for the (hadron) fermion which is $\widetilde{s u}(3)$ invariant as required. Indeed, as noted in [1], treating just the strong sector by itself, even after implementing the framon idea, one still would not obtain

any rotation. Only treating the electroweak and strong sectors together as in the present framework and implementing then the framon structure will one find that rotation of the fermion mass matrix results. It would thus seem that the rotation of the fermion mass matrix here is a consequence of the present framework produced from its very depths.

\section{Fermion Mixing and Mass Hierarchy}

In a nutshell, that fermion mixing and mass hierarchy would result from a rotating fermion mass matrix can be seen as follows. A mass matrix of the simple form (6.11) of a product of a vector $\boldsymbol{\alpha}$ with its hermitian conjugate which depends on the fermion species only through its normalization $m_{T}$ means of course that at any chosen value of the scale $\mu$, there is only 
one eigenvector with non-zero eigenvalue, namely $\boldsymbol{\alpha}$ with eigenvalue $m_{T}$, and that this vector, though not its eigenvalue, is the same for all fermion species. However, the masses and state vectors of physical particles are not measured all at the same scale. Indeed, the normal convention is to measure these quantities each at the scale equal to the particle mass itself. Thus, for example, the state vectors of the $t$ and $b$ quarks are to be taken respectively at $\mu=m_{t}$ and $\mu=m_{b}$, and being the heaviest state each in its own species, they are to be identified as the eigenstate of $m$ with nonzero eigenvalue, namely $\boldsymbol{\alpha}$, but one at $\mu=m_{t}$ and the other at $\mu=m_{b}$, thus:

$$
\mathbf{v}_{t}=\boldsymbol{\alpha}\left(\mu=m_{t}\right) ; \quad \mathbf{v}_{b}=\boldsymbol{\alpha}\left(\mu=m_{b}\right)
$$

Since the scales differ, however, and $\boldsymbol{\alpha}$ rotates with scale, it follows that $\mathbf{v}_{t}$ and $\mathbf{v}_{b}$ will no longer be aligned, or that the CKM matrix element:

$$
V_{t b}=\mathbf{v}_{t}^{*} \cdot \mathbf{v}_{b} \neq 1 \text {. }
$$

Hence mixing between the $U$ and $D$ states. Further, the $c$ quark, being an independent quantum state to $t$, must have a state vector $\mathbf{v}_{c}$ orthogonal to $\mathbf{v}_{t}$. This means that at the scale $\mu=m_{t}$ where $\mathbf{v}_{t}$ is defined as the only eigenstate of $m$ with nonzero eigenvalue, $\mathbf{v}_{c}$ must be an eigenstate of $m$ with a zero eigenvalue. But this is not the mass $m_{c}$ of the $c$ quark which is to be measured instead at the scale $\mu=m_{c}$, at which scale the eigenvector $\boldsymbol{\alpha}$ with the nonzero eigenvalue would have rotated already to a different direction and no longer orthogonal to the state $\mathbf{v}_{c}$. At $\mu=m_{c}$, therefore, $\mathbf{v}_{c}$ can no longer be an eigenvector with zero eigenvalue but, having now a component in the direction of the massive state $\boldsymbol{\alpha}$, would have acquired a nonzero mass, as if by "leakage" from the heavy state. Hence the mass hierarchy. Details for working out mixing matrices and lower generation masses from a given rotating mass matrix can be found in [5, 8, 7, 4, in the context of our ealier phenomenological model, the DSM, or in a general context, and thus more lucid language, in a recent note [20].

That a rotating fermion mass matrix can give a reasonable description of the fermion mass hierarchy and of the mixing phenomena including neutrino oscillations observed in experiment has been demonstrated in a number of earlier articles [7, 4]. Indeed, it can even be said that the data already give circumstantial evidence for mass matrix rotation [16]. Further, an explicit model (DSM) was constructed which was able to reproduce most mass ratios and mixing angles to within present experimental errors starting from just a small number of fitted parameters. However, it was noted in [7], based on 
the experience gained from the fits, that the details of the model are not so crucial, but that other models with a rotating mass matrix of the form (6.11) with rotational fixed points at $\mu=0$ and $\mu=\infty$, and a few parameters to adjust, may quite likely do as well.

Very briefly, this can be seen as follows. In the picture with a rotating mass matrix outlined at the beginning of this section, both the masses of lower generation fermions and the mixing between up and down fermion states arise from the rotation. Hence, the slower the rotation with respect to the change of scale, the smaller will also be the resultant effects. Now suppose that given a model with the stated properties, one is able to choose its parameters so as to have the heavy fermions, such as $t$ and $b$, close to the high energy fixed point at $\mu=\infty$ and the very light neutrinos close to the low energy fixed point at $\mu=0$, while the remaining fermions with intermediate masses lie somewhere in between where the rotation is faster, then, apart from the general features of up-down mixing and mass hierarchy already noted, the following empirical facts will automatically result:

(i) $m_{c} / m_{t}<m_{s} / m_{b}<m_{\mu} / m_{\tau}$ by virtue of the relative proximity of $t, b, \tau$ in that order to the high energy fixed point so that the "leakage" of masses to the lower generation $c, s, \mu$ is increasing in that order.

(ii) The mixing between quarks is considerably smaller than that between leptons, by virtue of the heavier masses of the quarks which place them closer to the high energy fixed point than the leptons.

(iii) The corner elements of the mixing matrices, i.e. $V_{u b}$ and $V_{t d}$ in the CKM matrix for quarks, and $U_{e 3}$ in the MNS matrix for leptons, are much smaller than the other elements, by virtue of the geometrical fact that the corner elements are associated with the torsion but the others with the curvature of the rotation trajectory traced out by $\boldsymbol{\alpha}$ through changing scale [18].

(iv) The element $U_{\mu 3}$ which governs the oscillation of atmospheric neutrinos is near maximal by virtue of the neutrino's close proximity to the low energy fixed point at $\mu=0$.

These already encompass most salient features of the fermion mass and mixing patterns known experimentally to-date.

Furthermore, a scheme where lower generation fermion masses are obtained by "leakage" via a rotating factorizable mass matrix has the following added attraction. Since the mass matrix remains factorizable at all scales, 
it has always some zero eigenvalues although the actual fermion masses are nonzero, which means that the QCD phase angle $\theta$ can be rotated away and the strong $\mathrm{CP}$ problem avoided [20].

Now, the present framework differs from the old DSM considerably in its starting premises and in all its structural details. Nevertheless it has surprisingly retained a similarity with DSM as regards precisely those properties noted above as relevant for deriving the physical effects in mind. First, it has a degenerate vacuum depending on orientation as DSM had, which is the initial condition needed for rotation to take place. Then it is found to give quark and lepton mass matrices of the required factorized form which rotates as the scale changes, and that this rotation has a fixed point at $\mu=0$ and another at $\mu=\infty$. It seems thus quite probable, judging from past experience, that the present framework will give (or can be made to give by appropriate adjustments of parameters) similar agreement with experiment as DSM did, although whether this will indeed be the case has yet to be demonstrated by explicit calculations.

Assuming optimistically for the moment that this will turn out to be the case, let us examine what we have gained with the present framework in comparison with the old DSM [7, 4]. First, we have gained on the theoretical basis. The DSM was a phenomenological model constructed for the explicit purpose of understanding the generations phenomenon without too high a demand for theoretical consistency, and some of the assumptions made were a little ad hoc. In the present framework, on the other hand, we have chosen to start with just a few main assumptions, and then to work systematically through the consequences to their logical conclusion. Thus, while the present framework has the hope to be developed into a theory, the DSM would have to remain a phenomenological model.

Secondly, there have been major improvements too on specific points, both in principle and in practice. We list in particular the following, which serve also to highlight some intricate features of the present model we find attractive.

(a) One outstanding weakness of the old DSM is that the electroweak symmetry $u(1) \times s u(2)$ has never been properly incorporated. In the present framework, not only is the electroweak symmetry fully incorporated, but is treated according to the same general principles as $s u(3)$, the other local gauge symmetry. Thus all the local symmetries $u(1), s u(2), s u(3)$ are taken to remain exact, and both nonabelian symmetries to be confining, and all are associated through the framon idea with each a "dual" global symmetry, i.e. $\tilde{u}(1), \widetilde{s u}(2), \widetilde{s u}(3)$. While the global abelian symmetry $\tilde{u}(1)$ remains exact 
and gives rise to baryon-lepton conservation as a bonus, the nonabelian global symmetries are both broken. The breaking is different for $\widetilde{s u}(2)$ and $\widetilde{s u}(3)$, but this difference is not imposed by fiat but arises as a consequence of the difference in structure between $s u(2)$ and $s u(3)$ which allows the framons of $s u(2)$ to satisfy the stronger unitarity constraint (2.3) than that admissible for $s u(3)$ framons. This forces the "weak" framons $\phi^{\tilde{r}}, \tilde{r}=1,2$ in $s u(2)$ to have opposite $u(1)$ charges, hence breaking by their different $u(1)$ gauge interactions the $\widetilde{s u}(2)$ invariance. The "strong" framons, on the other hand, have all the same $u(1)$ charge. Hence, in contrast, $u(1)$ gauge interaction does not break $\widetilde{s u}(3)$ invariance. What breaks $\widetilde{s u}(3)$ invariance instead is the linkage term $\nu_{2}$ in the framon potential via the vector $\boldsymbol{\alpha}$ originating in $s u(2)$. One sees thus that it is ultimately the difference in basic structure of the 2 symmetries $s u(2)$ and $s u(3)$, and not the manner they are treated, which is responsible for the difference in physical outcome between the 2 cases.

(b) A second weakness of the old DSM is that the self-interaction potential of scalar fields is not unique. That potential was constructed on the basis of an assumed permutation symmetry between the frame vector fields, which though not unreasonable, is essentially phenomenological and ad hoc. Even then, the potential there is not the only one which can be constructed with this symmetry. In contrast, the framon potential (3.11) of the present scheme is constructed on the basis of the doubled invariance under both the original local gauge symmetries $u(1) \times s u(2) \times s u(3)$ and their associated global symmetries $\tilde{u}(1) \times \widetilde{s u}(2) \times \widetilde{s u}(3)$, an invariance embedded already in the framon idea. It is also the only potential that we have been able to construct with the prescribed invariance up to fourth order for renormalizability. This means that under radiative corrections the potential will remain of the same form, only changing the values of the 7 coupling parameters which appear there. Such will not be the case for the potential in DSM, which under radiative corrections can acquire new terms not yet included. Moreover, this unique framon potential of the present scheme has some very attractive properties. It has a part involving the "weak" framons only which is of the same form as that for the standard electroweak theory, and another part involving the "strong" framons only which is close to the potential of DSM constructed for its favourable phenomenological properties. What is most interesting, however, are the additional $\nu_{1}, \nu_{2}$ terms linking the "weak" and "strong" sectors which are automatically admitted and required by the invariance. These not only distort the "strong" sector vacuum in such a way as to make it partake even more of the phenomenologically favourable properties of the DSM potential, e.g. to make its vacuum degenerate and amenable to rotation, but 
also couple automatically the "weak" and "strong" sectors in such a way that this rotation originating in the "strong" sector is carried over into the "weak" sector where the quarks and leptons of primary interest occur.

(c) In DSM, there being no requirement of $\widetilde{s u}(3)$ invariance, the Yukawa term was constructed just by summing over the free $\tilde{a}$ indices so as to guarantee at least permutation symmetry, which we have always regarded as a somewhat weak assumption. Nevertheless, it was this assumption which led to the factorizable fermion mass matrix, a rather important requirement for the scheme to work. In the present scheme, one has instead the $\widetilde{s u}(3)$ invariance which is inherent already in the framon idea, and this allowed one to construct seemingly unique Yukawa terms for both the weak and strong sectors leading automatically to factorizable fermion matrices. In constructing the Yukawa term for the strong sector one had to rely on the vector $\boldsymbol{\alpha}_{0}$ coming from the weak sector to maintain $\widetilde{s u}(3)$ invariance, while for the weak sector one had to rely on the vector $\boldsymbol{\alpha}^{( \pm)}$coming from the electromagnetic sector to maintain $\widetilde{s u}(2)$ invariance. Interestingly, it is exactly this intricate interplay between the various symmetries imposed by the framon idea on the Yukawa terms and on the framon potential in (b) that has allowed one not only to keep nearly all of the DSM's phenomenologically favourable features but also, it seems, to avoid most of its pitfalls, as will be seen in the following examples.

(d) In DSM, as explained in (a), the electroweak sector was not properly incorporated, and no consideration was yet given to the confinement picture of symmetry breaking as set out in our assumption (B) in the introduction. In the present language when assumption (B) is adopted, the states we called quarks and leptons in DSM would now appear as bound states of the fundamental fermion fields with framons confined via colour $s u(3)$. In other words, they would be hadrons, not quasi-elementary states in what we now call the standard model scenario. Of course, one need not perhaps insist on the confinement picture, but this would seem now unnatural in view of its success in other circumstances. Instead, in the present scheme, quarks and leptons, appearing now as bound states via the supposedly much deeper su(2) confinement, would appear as quasi-elementary under present experimental conditions.

(e) There was a mystery in the DSM which was not resolved within its own context. In writing down the fermion mass matrix in a form analogous to (6.11) above, the vector, say $\boldsymbol{\alpha}^{\prime}$, which appeared there originated in the strong sector and could depend in principle, as functions of the scale $\mu$, on the fermion species, i.e. whether $U, D$, charged leptons, or neutrinos. However, 
to our surprise, in fitting data then [8] with these $\boldsymbol{\alpha}^{\prime}$ as parameters, it turned out that the best fits for the first 3 species were accurately identical. So much so, indeed, that for all subsequent fits, we have just taken the same $\boldsymbol{\alpha}^{\prime}$ for all species. This gives the, to us, very appealing picture of all fermions lying on the same rotation trajectory as depicted in Figure 3 of [7] or Figure 7 of [4], and as utilised implicitly in coming to the conclusions (i) - (iv) above. Why the trajectories for all $\boldsymbol{\alpha}^{\prime \prime}$ s should be the same, however, was not explained in DSM. Here, in contrast, as asserted in the preceding entry (b), the vector $\boldsymbol{\alpha}$ which appears in the quark and lepton mass matrix (6.11) originates in the weak framon and does not depend on the fermion species. The rotation mechanism, moreover, comes from the strong sector and gets transmitted to the vector $\boldsymbol{\alpha}$ only through the linkage term $\nu_{2}$ in the framon potential which contains no species dependence. In other words, that all fermion species have the same rotation trajectory, which was an empirical observation deduced from fitting experimental data in DSM, seems now to be an automatic prediction in the present framework. An analogous observation applies to the normalisation of $\boldsymbol{\alpha}$ which is here a matter of definition, whereas for its parallel $\boldsymbol{\alpha}^{\prime}$ in DSM, the normalisation changed as the scale changed and caused there some problems.

(f) One unhappy feature of DSM was the existence in the Higgs spectrum of 2 zero modes [8]. These arose in the old model as a direct consequence of the 2-dimensional degeneracy of the vacuum just mentioned, i.e. in addition to the degeneracy connected to the local gauge invariance of the system and cannot thus be eaten up by the vector bosons. These Higgs zero modes were a headache, for they carry generation indices but no (up-down) flavour index, i.e. charge, which meant that they could give rise to FCNC effects unsuppressed by a mass. Although in the cases we have examined [19], disaster was avoided by some special features in their couplings, they remained a major concern for the model. In the present framework, however, there are no corresponding zero modes. Recall the Higgs mass matrix given explicitly above in (5.11) for the 2-G model. There is no zero mode there although, given the analogous 1-dimensional degeneracy of the vacuum in the $2-\mathrm{G}$ model, one might expect one zero mode by analogy. The reason for this difference between the two schemes is a rather subtle one. In both, the degeneracy of the vacuum arises, of course, from a symmetry; not the local gauge symmetry, but in DSM from the permutation symmetry mentioned in (b) and in the present framework from the global $\widetilde{s u}(3)$ symmetry. The difference, however, lies in the fact that the symmetry index in the present framework is carried by the framon only in the global factor $\boldsymbol{\alpha}$ which is $x$-independent, as seen in 
(2.10), while in DSM it is carried by the $x$-dependent field itself. Now a field can fluctuate and give rise to Higgs bosons, but not an $x$-independent global factor like $\boldsymbol{\alpha}$; hence the difference. The absence of these zero modes in the present model has thus removed a big worry as regards possible violations of experimental FCNC bounds.

(g) In DSM, the rotating vector called $\boldsymbol{\alpha}^{\prime}$ in (e) above which gives the fermion mass hierarchy and mixing patterns is actually the vector $\mathbf{r}=(x, y, z)$ introduced before in (4.5), and this rotates from the high scale $(\mu=\infty)$ fixed point at $\mathbf{r}=(1,0,0)$ to the low scale $(\mu=0)$ fixed point at $\mathbf{r}=\frac{1}{\sqrt{3}}(1,1,1)$. To fit experimental data, this $\mathbf{r}$ has to go pretty much all the way between these 2 fixed points within the physical range from $\mu=m_{t}$ the top-quark mass, to $\mu=m_{\nu}$ the neutrino masses, i.e. the 14 orders of magnitude or so in energy accessed by present experiment. Embarrassingly, however, the gauge and Higgs boson masses in the model depend on the values of $\mathbf{r}$, with

some e.g. proportional to $\sqrt{y^{2}+z^{2}}$, and will thus vary greatly with scale when $\mathbf{r}$ varies over the above range. This fact puts serious constraints on the DSM's phenomenological applicability. In the present scheme, there is no parallel difficulty. Although the vector $\boldsymbol{\alpha}$ which appears in the quark and lepton mass matrices does vary over a similar range as $\mathbf{r}$ in DSM, which ought to be sufficient for fitting the same experimental data on fermion mass and mixing patterns, the quantities $x, y, z$ on which the boson masses depend never change much from one another, with differences $\Delta$ always less than $R$ which has supposedly a small value.

Besides, there are bonuses such as an explanation for baryon-lepton number conservation and the possibility of understanding CP-violation in mixing which was beyond the older model. Thus so long as the projection made before of the present scheme's viability in phenomenological application is confirmed, then one would end up with a much stronger framework both from the theoretical and from the practical standpoint.

\section{Concluding Remarks}

Let us begin by counting up what seem to have been gained:

(I) One has assigned to scalar fields a geometrical significance that they previously lacked.

(II) One has gained thereby a theoretical criterion on what scalar fields are to be introduced into a gauge theory and needs no longer rely entirely 
on the dictates of experiment.

(III) One has answered, or at least bypassed, the question why, of the 2 nonabelian symmetries in the Standard Model, one $(s u(2))$ is broken, but the other $(s u(3))$ is confined. One now asserts instead that all local gauge symmetries remain exact, and both nonabelian gauge symmetries are confining. It is only the global symmetries $\widetilde{s u}(2)$ and $\widetilde{s u}(3)$ arising by virtue of the framon idea in (I), which are broken.

(IV) One has gained a solution to the old puzzle of baryon number conservation (in its modern form of $B-L$ conservation). This turns out here to be the same as conservation of the $\tilde{u}(1)$ charge, again a consequence of the framon idea (I), which quarks and leptons, as bound states of $s u(2)$ confinement, have acquired from framons as one of their constituents.

(V) One has recovered the electroweak theory in its standard form.

(VI) One has acquired for quarks and leptons, again from framons through $s u(2)$ confinement, an index referring to the global symmetry $\widetilde{s u}(3)$ which can play the role of generations. So fermion generations is "dual" to colour as baryon-lepton number is to electric charge, or as up-down flavour is to the confining $s u(2)$.

(VII) One has reproduced, with a structurally very different but theoretically much more consistent scheme, the essential features of an earlier phenomenological model which was successful in explaining the mass and mixing patterns of leptons and quarks observed in experiments.

A key note in the theoretical construction leading to the above enumerated gains seems to be that of economy. Not only has no local gauge symmetry larger than that of the Standard Model been introduced and no extension of the Standard Model framework been made along popular lines as supersymmetry, higher space-time dimensions, or extended structures like strings and branes, but even within the present scheme itself, any ambiguities which arise (such as embeddings, representations etc.) have been consistently settled by an insistence on minimality, and in almost every case, nature seems to have agreed. It is not that the present scheme cannot admit of extensions by supersymmetry, high space-time dimensions or string-like or brane-like structures, for we can see no contradiction in principle of the present scheme with all those, but that for answering the questions posed at the beginning that interest us, there seems no need at present for such extensions. When 
approached as suggested here, the answers sought appear to be there already within the Standard Model framework.

Comparing now the list above to the questions posed at the beginning in the Introduction, one sees that that there is now an answer offered for most. There is even a bonus, namely a solution offered for the ancient puzzle why baryon (or baryon-lepton) number is conserved, which was not conceived as one of the original aims. But there remains one question unanswered and one to which the answer is incomplete. The latter is why the distinction seen in nature between the 2 nonabelian symmetries in the Standard Model. Although in the confining picture, as in (III) above, one has reduced the question by taking both $s u(2)$ and $s u(3)$ as confining, it still begs the question why one can assume, as one needs to do and as 't Hooft and Banks and Rabinovici did, that $s u(2)$ confinement is so much deeper than $s u(3)$ colour confinement as to be completely undetected at present. The other is why, as assumed here, and also in the conventional formulation of the Standard Model, that su(2) doublets are left-handed but su(2) singlets right-handed. In place of answers to these questions, we can offer at present only some, perhaps slightly wild, speculations which we are not as yet in a position to substantiate.

When the idea that fermion generations may be dual to colour was first conceived [5], it was actually envisaged that by duality here one meant the nonabelian generalisation of the electromagnetic or Hodge star duality, as proposed, for example, in [21], although at that stage, nothing concrete was made out of the proposal. As the idea is developed in the present paper, however, the "duality" between the local gauge symmetries and their global duals is really just a change of frames, sharing little of the intricacies of the Hodge star. For instance, the massive vector bosons $\tilde{B}_{\mu}$ in the electroweak theory as treated here and in [2, 3] is coupled with the same strength $g_{2}$ as the gauge boson $B_{\mu}$. In contrast, if $B_{\mu}$ and $\tilde{B}_{\mu}$ were really dual in the (generalised) Hodge-star sense, we would expect the 2 coupling strengths to be related by a Dirac quantisation relation, say:

$$
g_{2} \tilde{g}_{2}=4 \pi,
$$

with $g_{2}$ being the coupling strength for $B_{\mu}$ and $\tilde{g}_{2}$ that for $\tilde{B}_{\mu}$. Besides, if the (generalised) Hodge dual to $s u(2)$ exists, as [21] claims, then there is an old result of 't Hooft which says that the dual symmetry will be broken [2], meaning presumably that it will be associated with some massive vector bosons which will be coupled with strength $\tilde{g}_{2}$ and not $g_{2}$. Can we then be sure that the actual massive vector bosons $W-Z$ we presently see in 
experiment are the $\tilde{B}_{\mu}$ considered above, or are they the (generalised) Hodge duals here considered? Suppose for the moment that it is the latter we see, with the experimentally measured coupling strength of $\alpha_{2}=\tilde{g}_{2}^{2} / 4 \pi \sim 0.034$, then by the Dirac quantisation condition above, the coupling strength $g_{2}$ of the gauge boson $B_{\mu}$ would be very large, of the order 20, much larger than the coupling strength of the colour gluon, $g_{3} \sim 1.2$. One could perhaps then understand why $s u(2)$ confinement is so much deeper than $s u(3)$ colour confinement. Besides, if $g_{2}$ is really that large, then the massive vector bosons $\tilde{B}_{\mu}$ considered before whose mass is proportional to $g_{2}$ will be extremely heavy, probably much heavier than the (generalised) Hodge duals that we identify as the $W$ 's and $Z$. If so we would have answered why they are not seen, and hence the whole question that was posed. But at the moment, we have no clear idea whether this can indeed be the case.

Amusingly, the speculation aired in the preceding paragraph offers also a possible answer to another question posed before concerning the apparent lack of influence on physics at our present "low" energies from higher vector boson states which are expected as radial and orbital excitations of the $W, Z-\gamma$ complex when the confinement picture of the electroweak theory is taken to be physical. This lack is intuitively understandable, and is confirmed by analysis in [12, if the excited states are much heavier than the ground states $W, Z-\gamma$, but the question remains why the states $W, Z-\gamma$ should be so much lighter than their excitations, which seems in some conflict with our experience in the parallel scenario of colour $s u(3)$ confinement. However, if our assumption above makes sense, then the states $W, Z-\gamma$ are not just the lowest in a tower of radial and orbital excitations as pictured before, but a completely different object, namely the dual to the gauge bosons via a nonabelian generalization to Hodge duality. This latter is a very complicated state, in a sense containing already a complex mixture of radially and orbitally excited components and cannot easily admit, presumably, any further simple excitations. What corresponds instead to the ground states in the familiar tower of radial and orbital excitations are not then the $W, Z-\gamma$ states but the states called $\tilde{B}_{\mu}$ in the preceding paragraph, which, as the argument goes, are already very much higher in mass than the $W, Z-\gamma$ bosons we experimentally observe. And their excitations would be even higher. There is then little wonder that they have no observable influence on the "low" energy physics accessible to us at present.

Our speculative answer to the other question why su(2) doublets are lefthanded but singlets right-handed is perhaps little more than just wishful thinking. The point is that in the confinement picture, doublets have to be 
confined, and the actual left-handed quarks and leptons we see are actually compound states made up of fundamental fermions and framons, while the right-handed are still just fundamental fermions. Besides, it is only the lefthanded quarks and lepton which interact with the vector bosons $W^{ \pm}, \gamma-Z$. Now, suppose for some reason that the framons should carry with them the projection operator $\frac{1}{2}\left(1+\gamma_{5}\right)$, then in all the terms in the action we wrote down, only left-handed flavour doublets and right-handed flavour singlets would occur, and all the vector bosons, being bound states of $\phi^{\dagger} \phi$, will be left-handed. But we can see no reason at present why framons as frame vectors should carry with them the said projection operator.

However, even with these 2 theoretical questions still unanswered, one has made, it seems, quite some progress towards a protogenic model. But what about phenomenology? Although the present scheme, as shown, has reproduced all the essential features of our earlier phenomenological (DSM) model, and has therefore, as judged by previous experience, a good chance of reproducing the earlier model's phenomenological successes, this has to be confirmed by calculation, which has started but not yet given definite results. Optimistically, there might even be a chance of improving on the earlier results, given that there may now be a possibility of getting $\mathrm{CP}$-violation in the mixing matrices which was not possible for the earlier model.

Then there is a host of phenomenological questions to be examined on whether the present scheme may give rise to new phenomena violating present experimental bounds. This will be a lengthy process which will take time to sort out. One of the foremost question to examine, we think, would be whether the proposed existence of the "strong" framons would disturb the apparent agreement of standard QCD with experiment in, for example, the running with energy of $\alpha_{S}$, the strong coupling constant. A preliminary investigation says no, given that the contribution of the scalar framons to the $\beta$-function is very small, only $1 / 8$ of that from fermions [22, 23], but this has yet to be systematically confirmed.

Again, optimistically, one may ask whether, apart from explaining known effects and surviving existing bounds, the present scheme gives some new characteristic predictions testable by foreseeable experiments. As far as we can see at present, any quantitative prediction will have to await the conclusion of the calculations and investigations projected in the last two paragraphs. One outstanding qualitative prediction, however, which might be testable already when the LHC comes into operation, is the existence of internal structures for leptons and quarks which up to now have appeared point-like. This prediction, though present already in the confinement pic- 
ture for the standard electroweak theory as an alternative interpretation, has now become an apparent imperative in the present scheme for, as we recall, it is through $s u(2)$ confinement that quarks and leptons acquire from their framon constituents both their generation index and their baryon-lepton number. In principle, the internal structures of quarks and leptons can be detected by deep inelastic scattering experiments as the structure of the proton was detected, only at a much deeper level. Unfortunately, our meagre understanding of $s u(2)$ confinement at present is insufficient to predict at what depth the internal structure of quarks and leptons are to be detected. But for all we know, it may be just around the corner, and it would seem to pay the experimenter to look out for it as soon as the LHC starts to run.

The prediction of internal structure for quarks and leptons, though physically highly significant, is insufficiently special or specific to distinguish the present scheme from other composite models. However, the same dynamics that led here to the said internal structure can manifest itself also in the existence of excited quark and lepton states, as already mentioned, which may be experimentally produced if the energy is high enough. And the spectrum of these will depend on the details of the scheme. Indeed, if one takes the confinement picture of the electroweak theory as actually physical, then there would be a host of other new states formed by $s u(2)$ confinement from any singlet combinations of the fundamental framon, gauge and fermion fields. This will open up a whole new field of spectroscopy to future investigation at sufficiently high energy which is potentially every bit as rich as, if not even richer than, hadron spectroscopy.

Of most exotic interest in this scenario would perhaps be those states formed from a pair of the fundamental fermion fields $\psi(2,1)$ or $\psi(2,3)$, which (though bosonic) are analogous to baryons in hadron spectroscopy. They can be grouped into the following 3 types: $\psi(2,1) \psi(2,1), \psi(2,3) \psi(2,3)$, and $\psi(2,1) \psi(2,3)$, which, for reasons to be made clear, we shall label as dileptons, diquarks and lepto-quarks respectively. Generically, of course, dilepton, diquark, and lepto-quark states occur in any scheme where the confinement picture for $s u(2)$ is taken as physical, as already considered in for example [12. But in the present framework, there is a new twist, because of the particular way that the baryon-lepton number and the generation index are introduced. We recall that the baryon-lepton number as well as the generation index in the present scheme are attached not to the fundamental fermion fields but to the framon fields, and only got transmitted to the bound quark and lepton states via $s u(2)$ confinement of the fundamental fermion fields with the framons. We have thus the unusual situation that what were 
called dileptons, diquarks and lepto-quarks above, comprising as they did only fundamental fermion states, do not actually carry a baryon or lepton number, nor for that matter a generation index. Nevertheless, when they decay, as they presumably will since they are expected to have quite high masses, they would do so most probably, in analogy to what hadrons do, by creating a framon-antiframon pair which, by combining respectively with the 2 constituent fundamental fermion fields, will give back the appropriate baryon-lepton numbers and generation indices to the quarks and leptons in the decay products. Hence, the first type will decay into a pair of leptons, the second into a pair of quarks, and the third into a lepton and a quark, as the labels of respectively dileptons, diquarks and lepto-quarks suggest. However, the spectrum of of these states will be different in the present framework from that obtained in models of $s u(2)$ confinement [12] where the baryonlepton number and generation index are attached instead to the fundamental fermion fields. Thus, given sufficiently high energy for this new spectroscopy to be explored, there should, we think, be no great difficulty in distinguishing the present framework from the others. Clearly, a lot of the details of the phenomenology remain yet to be worked out, the treatment of which would take us way beyond the scope of the present paper, and has to be left to be supplied, we hope, elsewhere later. But the brief discussion above may already serve to indicate the richness of this possible new field of particle spectroscopy, which can become a prolific hunting ground for future experiments, if not yet already at LHC, then one day when high enough energies are available.

Lastly, we note that of the physical ingredients making up the present framework, the most distinctive are perhaps the strong framon fields $\phi_{a}^{\tilde{a} \tilde{r}}$, at least when taken as elementary as they are in this paper. It would be nice, therefore, if one could device a phenomenological handle for their detection. These are colour triplets and can thus, like quarks, manifest themselves in 2 ways, either confined into freely propagating colour singlet hadron states, or else appearing as jets in hard scattering, but the detection of either will not be altogether easy. For example, by binding together a framon-antiframon pair, we would obtain in the $s$-wave the scalar states studied in the second half of section 5 , or else in the $p$-wave some analogous vector states. But these are hadrons with presumably rather high masses, and hence broad widths, and will not be easy either to detect or to distinguish at first sight from hadrons of the common $q \bar{q}$ type. Nevertheless, we do know a fair amount about these states, as the analysis of section 5 shows, and this can be further extended, so that with luck and hard work, sufficient distinctive features may 
be identified for their eventual detection. The same remark applies to the problem of detecting the jets in hard scattering originating from framons and distinguishing them from the quark jets also produced. Clearly, there is scope for much future phenomenology in this direction.

We are much indebted for constant interest and encouragement as well as for occasional remarks and help in checking certain arguments to José Bordes who has been in fact our collaborator in spirit throughout the several long years that this work has occupied us.

\section{References}

[1] Chan Hong-Mo and Tsou Sheung Tsun, "Higgs Fields as Vielbeins in Internal Symmetry Space", hep-ph/0611363.

[2] G. 't Hooft, Acta Phys. Austr., Suppl. 22 (1980) 531

[3] T. Banks and E. Rabinovici, Nucl. Phys. B160 (1979) 347.

[4] Chan Hong-Mo and Tsou Sheung Tsun, Acta Phys. Polonica, 33 (2002) 4041, hep-ph/0303010.

[5] Chan Hong-Mo and Tsou Sheung Tsun, Phys. Rev. D57 (1998) 2507, hep-th/9701120.

[6] G. 't Hooft, Erice lecture notes Aug/Sept 1998, hep-th/9812204 v2 (1999).

[7] José Bordes, Chan Hong-Mo and Tsou Sheung Tsun, Eur. Phys. J. C. 10, 63 (1999), hep-ph/9901440.

[8] José Bordes, Chan Hong-Mo, Jacqueline Faridani, Jakov Pfaudler, and Tsou Sheung Tsun, Phys. Rev. D58, 013004, (1998), hep-ph/9712276.

[9] See e.g. Chan Hong-Mo and Tsou Sheung Tsun, Some Elementary Gauge Theory Concepts, World Scientific, Singapore, 1993.

[10] L.F. Abbott and E. Farhi, Phys. Lett. 101B (1981) 69.

[11] L.F. Abbott and E. Farhi, Nucl. Phys. B189 (1981) 547.

[12] M. Claudson, E. Farhi, and R.L. Jaffe, Phys. Rev. D34 (1986) 873. 
[13] For an example of a recent work in this direction, see e.g. Xavier Calmet and Harald Fritzsch, Phys. Lett. B496 (2000) 161, hep-ph/0008243v2.

[14] T.D. Lee and C.N. Yang, Phys. Rev. 98 (1955) 1501.

[15] Steven Weinberg, Phys. Rev. D7, (1973) 2887.

[16] José Bordes, Chan Hong-Mo and Tsou Sheung Tsun, Eur. Phys. J. C 27 (2003) 189-200, hep-ph/0203124.

[17] José Bordes, Chan Hong-Mo and Tsou Sheung Tsun, Phys. Rev. D65, 093006, (2002), hep-ph/0111369.

[18] José Bordes, Chan Hong-Mo, Jakov Pfaudler, and Tsou Sheung Tsun, Phys. Rev. D58, 053006, (1998), hep-ph/9802436.

[19] José Bordes, Chan Hong-Mo, Jacqueline Faridani, Jakov Pfaudler, and Tsou Sheung Tsun, Phys. Rev. D60, 013005, (1999), hep-ph/9807277.

[20] José Bordes, Chan Hong-Mo and Tsou Sheung Tsun, arXiv:0707.3358.

[21] Chan Hong-Mo, J. Faridani and Tsou Sheung Tsun, Phys. Rev. D53 (1996) 7293.

[22] David Gross and Frank Wilczek, Phys. Rev. Letters, 30, 1343 (1973).

[23] T. P. Cheng, E. Eichten and Ling-Fong Li, Phys. Rev. D9, 2259 (1974). 